\title{
LEBESGUE INEQUALITIES FOR CHEBYSHEV THRESHOLDING GREEDY ALGORITHMS
}

\author{
P. M. BERNÁ, Ó. BLASCO, G. GARRIGÓS, E. HERNÁNDEZ, AND T. OIKHBERG
}

\begin{abstract}
We establish estimates for the Lebesgue parameters of the Chebyshev Weak Thresholding Greedy Algorithm in the case of general bases in Banach spaces. These generalize and slightly improve earlier results in [9], and are complemented with examples showing the optimality of the bounds. Our results also correct certain bounds recently announced in [18], and answer some questions left open in that paper.
\end{abstract}

\section{INTRODUCTION}

Let $\mathbb{X}$ be a Banach space over $\mathbb{K}=\mathbb{R}$ or $\mathbb{C}$, let $\mathbb{X}^{*}$ be its dual space, and consider a system $\left\{\mathbf{e}_{n}, \mathbf{e}_{n}^{*}\right\}_{n=1}^{\infty} \subset \mathbb{X} \times \mathbb{X}^{*}$ with the following properties:
a) $0<\inf _{n}\left\{\left\|\mathbf{e}_{n}\right\|,\left\|\mathbf{e}_{n}^{*}\right\|\right\} \leq \sup _{n}\left\{\left\|\mathbf{e}_{n}\right\|,\left\|\mathbf{e}_{n}^{*}\right\|\right\}<\infty$
b) $\mathbf{e}_{n}^{*}\left(\mathbf{e}_{m}\right)=\delta_{n, m}$, for all $n, m \geq 1$
c) $\mathbb{X}=\overline{\operatorname{span}\left\{\mathbf{e}_{n}: n \in \mathbb{N}\right\}}$
d) $\mathbb{X}^{*}=\overline{\operatorname{span}\left\{\mathbf{e}_{n}^{*}: n \in \mathbb{N}\right\}}{ }^{*}$.

Under these conditions $\mathscr{B}=\left\{\mathbf{e}_{n}\right\}_{n=1}^{\infty}$ is called a seminormalized Markushevich basis for $\mathbb{X}$ (or M-basis for short), with dual system $\left\{\mathbf{e}_{n}^{*}\right\}_{n=1}^{\infty}$. Sometimes we shall consider the following special cases

e) $\mathscr{B}$ is a Schauder basis if $K_{b}:=\sup _{N}\left\|S_{N}\right\|<\infty$, where $S_{N} x:=\sum_{n=1}^{N} \mathbf{e}_{n}^{*}(x) \mathbf{e}_{n}$ is the $N$-th partial sum operator

f) $\mathscr{B}$ is a Cesàro basis if $\sup _{N}\left\|F_{N}\right\|<\infty$, where $F_{N}:=\frac{1}{N} \sum_{n=1}^{N} S_{n}$ is the $N$-th Cesàro operator. In this case we use the constant

$$
\beta=\max \left\{\sup _{N}\left\|F_{N}\right\|, \sup _{N}\left\|I-F_{N}\right\|\right\} .
$$

With every $x \in \mathbb{X}$, we shall associate the formal series $x \sim \sum_{n=1}^{\infty} \mathbf{e}_{n}^{*}(x) \mathbf{e}_{n}$, where a)-c) imply that $\lim _{n} \mathbf{e}_{n}^{*}(x)=0$. As usual, we denote $\operatorname{supp} x=\left\{n \in \mathbb{N}: \mathbf{e}_{n}^{*}(x) \neq 0\right\}$.

We recall standard notions about (weak) greedy algorithms; see e.g. the texts [22, 24] for details and historical background. Fix $t \in(0,1]$. We say that $A$ is a $t$-greedy set for $x$ of

1991 Mathematics Subject Classification. 41A65, 41A46, 46B15.

Key words and phrases. thresholding Chebyshev greedy algorithm, thresholding greedy algorithm, quasigreedy basis, semi-greedy bases.

The research of the first, third and fourth authors are partially supported by the grants MTM-2016-76566-P (MINECO, Spain) and 19368/PI/14 (Fundación Séneca, Región de Murcia, Spain). Also, the first author is supported by a PhD Fellowship from the program "Ayudas para contratos predoctorales para la formación de doctores 2017" (MINECO, Spain). The second author is supported by Grant MTM-2014-53009-P(MINECO, Spain). Third author partially supported by grant MTM2017-83262-C2-2-P (Spain). The fourth author has received funding from the European Union's Horizon 2020 research and innovation programme under the Marie Skłodowska-Curie grant agreement No 777822. 
order $m$, denoted $A \in G(x, m, t)$, if $|A|=m$ and

$$
\min _{n \in A}\left|\mathbf{e}_{n}^{*}(x)\right| \geq t \cdot \max _{n \notin A}\left|\mathbf{e}_{n}^{*}(x)\right| .
$$

A $t$-greedy operator of order $m$ is any mapping $\mathscr{G}_{m}^{t}: \mathbb{X} \rightarrow \mathbb{X}$ which at each $x \in \mathbb{X}$ takes the form

$$
\mathscr{G}_{m}^{t}(x)=\sum_{n \in A} \mathbf{e}_{n}^{*}(x) \mathbf{e}_{n}, \quad \text { for some set } \quad A=A\left(x, \mathscr{G}_{m}^{t}\right) \in G(x, m, t) .
$$

We write $\mathbb{G}_{m}^{t}$ for the set of all $t$-greedy operators of order $m$. The approximation scheme which assigns a sequence $\left\{\mathscr{G}_{m}^{t}(x)\right\}_{m=1}^{\infty}$ to each vector $x \in \mathbb{X}$ is called a Weak Thresholding Greedy Algorithm (WTGA), see [15, 23]. When $t=1$ one just says Thresholding Greedy Algorithm (TGA), and drops the super-index $t$, that is $\mathscr{G}_{m}^{1}=\mathscr{G}_{m}$, etc.

It is standard to quantify the efficiency of these algorithms, among all possible $m$-term approximations, in terms of Lebesgue-type inequalities. That is, for each $m=1,2, \ldots$, we look for the smallest constant $\mathbf{L}_{m}^{t}$ such that

$$
\left\|x-\mathscr{G}_{m}^{t}(x)\right\| \leq \mathbf{L}_{m}^{t} \sigma_{m}(x), \quad \forall x \in \mathbb{X}, \quad \forall \mathscr{G}_{m}^{t} \in \mathbb{G}_{m}^{t},
$$

where

$$
\sigma_{m}(x):=\inf \left\{\left\|x-\sum_{n \in B} b_{n} \mathbf{e}_{n}\right\|: b_{n} \in \mathbb{K}, \quad|B| \leq m\right\} .
$$

We call the number $\mathbf{L}_{m}^{t}$ the Lebesgue parameter associated with the WTGA, and we just write $\mathbf{L}_{m}$ when $t=1$. We refer to [24, Chapter 3] for a survey on such inequalities, and to [11, 9, 1, 4, 5] for recent results. It is known that $\mathbf{L}_{m}^{t}=O(1)$ holds for a fixed $t$ if and only if it holds for all $t \in(0,1]$, and if and only if $\mathscr{B}$ is unconditional and democratic; see [14] and [22, Thm 1.39]. In this special case $\mathscr{B}$ is called a greedy basis.

In this paper we shall be interested in Chebyshev thresholding greedy algorithms. These were introduced by Dilworth, Kalton and Kutzarova, see [7, §3], as an enhancement of the TGA. Here, we use the weak version considered in [9]. Namely, for fixed $t \in(0,1]$ we say that $\mathfrak{C G}_{m}^{t}: \mathbb{X} \rightarrow \mathbb{X}$ is a Chebyshev $t$-greedy operator of order $m$ if for every $x \in \mathbb{X}$ the set $A=\operatorname{supp} \mathfrak{C G}_{m}^{t}(x) \in G(x, m, t)$ and moreover

$$
\left\|x-\mathfrak{C G}_{m}^{t}(x)\right\|=\min \left\{\left\|x-\sum_{n \in A} a_{n} \mathbf{e}_{n}\right\|: a_{n} \in \mathbb{K}\right\} .
$$

Finally, we define the weak Chebyshevian Lebesgue parameter $\mathbf{L}_{m}^{\text {ch,t }}$ as the smallest constant such that

$$
\left\|x-\mathfrak{C G}_{m}^{t}(x)\right\| \leq \mathbf{L}_{m}^{\mathrm{ch}, t} \sigma_{m}(x), \quad \forall x \in \mathbb{X}, \quad \forall \mathfrak{C G}_{m} \in \mathbb{G}_{m}^{\mathrm{ch}, t},
$$

where $\mathbb{G}_{m}^{\text {ch,t }}$ is the collection of all Chebyshev $t$-greedy operators of order $m$. As before, when $t=1$ we shall omit the index $t$, that is $\mathbf{L}_{m}^{\mathrm{ch}}:=\mathbf{L}_{m}^{\mathrm{ch}, 1}$.

When $\mathbf{L}_{m}^{\text {ch }}=O(1)$ the system $\mathscr{B}$ is called semi-greedy; see [7]. We remark that the first author recently established that a Schauder basis $\mathscr{B}$ is semi-greedy if and only if is quasigreedy and democratic; see [2].

In this paper we shall be interested in quantitative bounds of $\mathbf{L}_{m}^{\mathrm{ch}, t}$ in terms of the quasigreedy and democracy parameters of a general M-basis $\mathscr{B}$. Earlier bounds were obtained by Dilworth, Kutzarova and Oikhberg in [9] when $\mathscr{B}$ is a quasi-greedy basis, and very recently, some improvements were also announced by C. Shao and P. Ye in [18, Theorem 
3.5]. Unfortunately, various arguments in the last paper seem not to be correct, so one of our goals here is to give precise statements and proofs for the results in [18], and also settle some of the questions which are left open there.

To state our results, we recall the definitions of the involved parameters. Given a finite set $A \subset \mathbb{N}$, we shall use the following standard notation for the indicator sums:

$$
\mathbf{1}_{A}=\sum_{n \in A} \mathbf{e}_{n} \quad \text { and } \quad \mathbf{1}_{\varepsilon A}=\sum_{n \in A} \varepsilon_{n} \mathbf{e}_{n}, \quad \varepsilon \in \Upsilon
$$

where $\Upsilon$ is the set of all $\varepsilon=\left\{\varepsilon_{n}\right\}_{n} \subset \mathbb{K}$ with $\left|\varepsilon_{n}\right|=1$. Similarly, we write

$$
P_{A}(x)=\sum_{n \in A} \mathbf{e}_{n}^{*}(x) \mathbf{e}_{n} .
$$

The relevant parameters for this paper are the following:

- Conditionality parameters:

$$
k_{m}:=\sup _{|A| \leq m}\left\|P_{A}\right\| \quad \text { and } \quad k_{m}^{c}=\sup _{|A| \leq m}\left\|I-P_{A}\right\| .
$$

- Quasi-greedy parameters:

$$
g_{m}:=\sup _{\mathscr{G}_{k} \in \mathbb{G}_{k}, k \leq m}\left\|\mathscr{G}_{k}\right\| \text { and } g_{m}^{c}:=\sup _{\mathscr{G}_{k} \in \mathbb{G}_{k}, k \leq m}\left\|I-\mathscr{G}_{k}\right\| .
$$

Below we shall also use the variant

$$
\tilde{g}_{m}:=\sup _{\substack{\mathscr{G} \prime<\mathscr{G} \\ \mathscr{G} \in \mathbb{G}_{k}, k \leq m}}\left\|\mathscr{G}-\mathscr{G}^{\prime}\right\|,
$$

where $\mathscr{G}^{\prime}<\mathscr{G}$ means that $A\left(x, \mathscr{G}^{\prime}\right) \subset A(x, \mathscr{G})$ for all $x$; see [4].

- Super-democracy parameters:

$$
\tilde{\mu}_{m}=\sup _{\substack{|A|=|B| \leq m \\|\varepsilon|=|\eta|=1}} \frac{\left\|\mathbf{1}_{\varepsilon A}\right\|}{\left\|\mathbf{1}_{\eta B}\right\|} \quad \text { and } \quad \tilde{\mu}_{m}^{d}=\sup _{\substack{|A|=|B| \leq m, A \cap B=\emptyset \\|\varepsilon|=|\eta|=1}} \frac{\left\|\mathbf{1}_{\varepsilon A}\right\|}{\left\|\mathbf{1}_{\eta B}\right\|} .
$$

- Quasi-greedy parameters for constant coefficients (see [4, (3.11)])

$$
\gamma_{m}=\sup _{\substack{|\varepsilon|=1 \\ B \subset A,|A| \leq m}} \frac{\left\|\mathbf{1}_{\varepsilon B}\right\|}{\left\|\mathbf{1}_{\varepsilon A}\right\|}
$$

Note that $\gamma_{m} \leq g_{m} \leq \tilde{g}_{m} \leq 2 g_{m}$, but in general $\gamma_{m}$ may be much smaller than $g_{m}$; see e.g. [4, §5.5]. Likewise, in $\S 5$ below we show that $\tilde{\mu}_{m}^{d}$ may be much smaller than $\tilde{\mu}_{m}$, except for Schauder bases in which both quantities turn out to be equivalent; see Theorem 5.2 .

Our first result is a general upper bound, which improves and extends [18, Theorem 2.4].

Theorem 1.1. Let $\mathscr{B}$ be an M-basis in $\mathbb{X}$, and let $\mathfrak{K}=\sup _{n, j}\left\|\mathbf{e}_{n}^{*}\right\|\left\|\mathbf{e}_{j}\right\|$. Then,

$$
\mathbf{L}_{m}^{\mathrm{ch}, t} \leq 1+\left(1+\frac{1}{t}\right) \mathfrak{K} m, \quad \forall m \in \mathbb{N}, t \in(0,1] .
$$

Moreover, there exists a pair $(\mathbb{X}, \mathscr{B})$ where the equality is attained for all $m$ and $t$.

The second result is a slight generalization of [9, Theorem 4.1], and gives a correct version of [18, Theorem 3.5]. 
Theorem 1.2. Let $\mathscr{B}$ be an $M$-basis in $\mathbb{X}$. Then, for all $m \geq 1$ and $t \in(0,1]$,

$$
\mathbf{L}_{m}^{\mathrm{ch}, t} \leq g_{2 m}^{c}+\frac{2}{t} \min \left\{\tilde{g}_{m} \tilde{\mu}_{m}, \gamma_{2 m} \tilde{g}_{2 m} \tilde{\mu}_{m}^{d}\right\}
$$

Our next result concerns lower bounds for $\mathbf{L}_{m}^{\mathrm{ch}, t}$, for which we need to introduce weaker versions of the democracy parameters with an additional separation condition. For two finite sets $A, B \subset \mathbb{N}$ and $c \geq 1$, the notation $A>c B$ will stand for $\min A>c \max B$.

- Given an integer $c \geq 2$, we define

$$
\vartheta_{m, c}:=\sup \left\{\frac{\left\|\mathbf{1}_{\varepsilon A}\right\|}{\left\|\mathbf{1}_{\eta B}\right\|}:|\varepsilon|=|\eta|=1,|A|=|B| \leq m \text { with } A>c B \text { or } B>c A\right\} \text {. }
$$

Theorem 1.3. If $\mathscr{B}$ is a Cesàro basis in $\mathbb{X}$ with constant $\beta$, then for every $c \geq 2$

$$
\mathbf{L}_{m}^{\mathrm{ch}, t} \geq \frac{1}{t \beta^{2}} \frac{c-1}{c+1} \vartheta_{m, c}, \quad \forall m \in \mathbb{N}, t \in(0,1] .
$$

We shall also establish, in Theorem 3.8 below, a similar lower bound valid for more general M-bases (not necessarily of Cesàro type), in terms of a new parameter $\theta_{m}$ which is invariant under rearrangements of $\mathscr{B}$.

Remark 1.4. One may compare the bounds for $\mathbf{L}_{m}^{\mathrm{ch}}$ above with those for $\mathbf{L}_{m}$ given in [4]

$$
\text { (1) } \mathbf{L}_{m} \leq 1+3 \mathfrak{K} m, \quad \text { (2) } \mathbf{L}_{m} \leq k_{2 m}^{c}+\tilde{g}_{m} \tilde{\mu}_{m}, \quad \text { and } \quad \text { (3) } \mathbf{L}_{m} \geq \tilde{\mu}_{m}^{d} \text {, }
$$

which illustrate a slightly better behavior of the Chebishev TGA. Observe that one also has the trivial inequalities

$$
\mathbf{L}_{m}^{\mathrm{ch}, t} \leq \mathbf{L}_{m}^{t} \leq k_{m}^{c} \mathbf{L}_{m}^{\mathrm{ch}, t}
$$

Indeed, $\mathbf{L}_{m}^{\text {ch }, t} \leq \mathbf{L}_{m}^{t}$ is direct by definition, while $\mathbf{L}_{m}^{t} \leq k_{m}^{c} \mathbf{L}_{m}^{\text {ch,t }}$ can be proved as follows: take $x \in \mathbb{X}$ and $A=\operatorname{supp} \mathscr{G}_{m}^{t}(x)$. Pick a Chebyshev greedy operator $\mathfrak{C G}_{m}^{t}$ such that supp $\mathfrak{C G}_{m}^{t}(x)=$ $A$. Then

$$
\left\|x-\mathscr{G}_{m}^{t}(x)\right\|=\left\|\left(I-P_{A}\right) x\right\|=\left\|\left(I-P_{A}\right)\left(x-\mathfrak{C G}_{m}^{t}(x)\right)\right\| \leq k_{m}^{c}\left\|x-\mathfrak{C G}_{m}^{t}(x)\right\|,
$$

so $\mathbf{L}_{m}^{t} \leq k_{m}^{c} \mathbf{L}_{m}^{\text {ch, } t}$. Hence, when $\mathscr{B}$ is unconditional then $\mathbf{L}_{m}^{t} \approx \mathbf{L}_{m}^{\text {ch, } t}$. However for all conditional quasi-greedy and democratic bases we have $\mathbf{L}_{m}^{\text {ch }}=O(1)$, but $\mathbf{L}_{m} \rightarrow \infty$.

The paper is organized as follows. Section 2 is devoted to preliminary lemmas. In Section 3 we prove Theorems 1.1, 1.2 and 1.3, and also establish the more general lower bound in Theorem 3.8, giving various situations in which it applies. Section 4 is devoted to examples illustrating the optimality of the results; in particular, an optimal bound of $\mathbf{L}_{m}^{\text {ch }}$ for the trigonometric system in $L^{1}(\mathbb{T})$, settling a question left open in [18]. In Section [5 we investigate the equivalence between $\tilde{\mu}_{m}^{d}$ and $\tilde{\mu}_{m}$ and show Theorem 5.2. Finally, in Section 6 we study the convergence of $\mathfrak{C G}_{m}(x)$ and $\mathscr{G}_{m}(x)$ to $x$ under the strong M-basis assumption, settling a gap in [18, 27].

\section{Preliminary Results}

We recall some basic concepts and results that will be used later in the paper; see [7, 4]. For each $\alpha>0$ we define the $\alpha$-truncation of a scalar $y \in \mathbb{K}$ as

$$
T_{\alpha}(y)=\alpha \operatorname{sign} y \text { if }|y| \geq \alpha, \quad \text { and } \quad T_{\alpha}(y)=y \text { if }|y| \leq \alpha
$$


We extend $T_{\alpha}$ to an operator in $\mathbb{X}$ by formally assigning $T_{\alpha}(x) \sim \sum_{n=1}^{\infty} T_{\alpha}\left(\mathbf{e}_{n}^{*}(x)\right) \mathbf{e}_{n}$, that is

$$
T_{\alpha}(x):=\alpha \mathbf{1}_{\varepsilon \Lambda_{\alpha}(x)}+\left(I-P_{\Lambda_{\alpha}(x)}\right)(x),
$$

where $\Lambda_{\alpha}(x)=\left\{n:\left|\mathbf{e}_{n}^{*}(x)\right|>\alpha\right\}$ and $\varepsilon=\left\{\operatorname{sign}\left(\mathbf{e}_{n}^{*}(x)\right)\right\}$. Of course, this operator is well defined since $\Lambda_{\alpha}(x)$ is a finite set. In [4] we can find the following result:

Lemma 2.1. [4, Lemma 2.5] For all $\alpha>0$ and $x \in \mathbb{X}$, we have

$$
\left\|T_{\alpha}(x)\right\| \leq g_{\left|\Lambda_{\alpha}(x)\right|}^{c}\|x\| \text {. }
$$

We also need a well known property from [7, 8], formulated as follows.

Lemma 2.2. [4, Lemma 2.3] If $x \in \mathbb{X}$ and $\varepsilon=\left\{\operatorname{sign}\left(\mathbf{e}_{n}^{*}(x)\right)\right\}$, then

$$
\min _{n \in G}\left|\mathbf{e}_{n}^{*}(x)\right|\left\|\mathbf{1}_{\varepsilon G}\right\| \leq \tilde{g}_{|G|} \mid\|x\|, \quad \forall G \in G(x, m, 1) .
$$

The following version of (2.1), valid even if $G$ is not greedy, improves [9, Lemma 2.2].

Lemma 2.3. Let $x \in \mathbb{X}$ and $\varepsilon=\left\{\operatorname{sign}\left(\mathbf{e}_{n}^{*}(x)\right)\right\}$. For every set finite $A \subset \mathbb{N}$, if $\alpha=\min _{n \in A}\left|\mathbf{e}_{n}^{*}(x)\right|$, then

$$
\alpha\left\|\mathbf{1}_{\varepsilon A}\right\| \leq \gamma_{\left|A \cup \Lambda_{\alpha}(x)\right|} \tilde{g}_{\left|A \cup \Lambda_{\alpha}(x)\right|}\|x\|,
$$

where $\Lambda_{\alpha}(x)=\left\{n:\left|\mathbf{e}_{n}^{*}(x)\right|>\alpha\right\}$.

Proof. Call $G=A \cup \Lambda_{\alpha}(x)$, and notice that it is a greedy set for $x$. Then,

$$
\alpha\left\|\mathbf{1}_{\varepsilon A}\right\| \leq \alpha \gamma_{|G|}\left\|\mathbf{1}_{\varepsilon G}\right\| \leq \gamma_{|G|} \tilde{g}_{|G|}\|x\|,
$$

using (2.1) in the last step.

Remark 2.4. The following is a variant of (2.2) with a different constant

$$
\min _{n \in A}\left|\mathbf{e}_{n}^{*}(x)\right|\left\|\mathbf{1}_{\varepsilon A}\right\| \leq k_{|A|}\|x\| .
$$

A similar proof as the one in Lemma2.3 can be seen in [3, Proposition 2.5].

Finally, using convexity as in [4, Lemma 2.7], one has the elementary lemma.

Lemma 2.5. For all finite sets $A \subset \mathbb{N}$ and scalars $a_{n} \in \mathbb{K}$ it holds

$$
\left\|\sum_{n \in A} a_{n} \mathbf{e}_{n}\right\| \leq \max _{n \in A}\left|a_{n}\right| \sup _{|\varepsilon|=1}\left\|\mathbf{1}_{\varepsilon A}\right\| .
$$

\section{PRoof of The MAIN RESUltS}

3.1. Proof of Theorem 1.1. Let $x \in \mathbb{X}$ and $\mathfrak{C G}_{m}^{t} \in \mathbb{G}_{m}^{\text {ch,t }}$ be a fixed Chebyshev $t$-greedy operator, and denote by $A=\operatorname{supp} \mathfrak{C G}_{m}^{t} x \in G(x, m, t)$. Pick any $z=\sum_{n \in B} b_{n} \mathbf{e}_{n}$ such that $|B|=m$. By definition of the Chebyshev operators,

$$
\left\|x-\mathfrak{C G}_{m}^{t}(x)\right\| \leq\left\|x-P_{A \cap B}(x)\right\| \leq\left\|P_{B \backslash A}(x)\right\|+\left\|x-P_{B}(x)\right\| .
$$

On the one hand, using (1.2),

$$
\left\|P_{B \backslash A}(x)\right\| \leq \sup _{n}\left\|\mathbf{e}_{n}\right\| \sum_{j \in B \backslash A}\left|\mathbf{e}_{j}^{*}(x)\right| \leq \frac{1}{t} \sup _{n}\left\|\mathbf{e}_{n}\right\| \sum_{j \in A \backslash B}\left|\mathbf{e}_{j}^{*}(x-z)\right| \leq \frac{1}{t} \mathfrak{K} m\|x-z\| .
$$

On the other hand, using the inequality (3.9) of [4],

$$
\left\|x-P_{B}(x)\right\|=\left\|\left(I-P_{B}\right)(x-z)\right\| \leq k_{m}^{c}\|x-z\| \leq(1+\mathfrak{K} m)\|x-z\| .
$$

Hence, $\mathbf{L}_{m}^{\mathrm{ch}, t} \leq 1+\left(1+\frac{1}{t}\right) \mathfrak{K} m$. Finally, the fact that the equality in (1.4) can be attained is witnessed by Examples 4.1 and 4.2 below. 
3.2. Proof of Theorem 1.2. The scheme of the proof follows the lines in [7, Theorem 3.2] and [9, Theorem 4.1], with some additional simplifications introduced in [4].

Given $x \in \mathbb{X}$ and $\mathfrak{C G}_{m}^{t} \in \mathbb{G}_{m}^{\text {ch,t }}$, we denote by $A=\operatorname{supp} \mathfrak{C G}_{m}^{t} x \in G(x, m, t)$. Pick any $z=\sum_{n \in B} b_{n} \mathbf{e}_{n}$ such that $|B|=m$. By definition of the Chebyshev operators,

$$
\left\|x-\mathfrak{C G}_{m}^{t} x\right\| \leq\|x-p\|, \quad \text { for any } p=\sum_{n \in A} a_{n} \mathbf{e}_{n} .
$$

We make the selection of $p$ suggested in [7]. Namely, if $\alpha=\max _{n \notin A}\left|\mathbf{e}_{n}^{*}(x)\right|$, we let

$$
p=P_{A}(x)-P_{A}\left(T_{\alpha}(x-z)\right) \text {. }
$$

It is easily verified that

$$
\begin{aligned}
x-p & =\left(I-P_{A}\right)\left(x-T_{\alpha}(x-z)\right)+T_{\alpha}(x-z) \\
& =P_{B \backslash A}\left(x-T_{\alpha}(x-z)\right)+T_{\alpha}(x-z) .
\end{aligned}
$$

Since $\Lambda_{\alpha}(x-z)=\left\{n:\left|\mathbf{e}_{n}^{*}(x-z)\right|>\alpha\right\} \subset A \cup B$, then Lemma2.1 gives

$$
\left\|T_{\alpha}(x-z)\right\| \leq g_{2 m}^{c}\|x-z\| \text {. }
$$

Next we treat the first term in (3.2). Observe that $\max _{n \in B \backslash A}\left|\mathbf{e}_{n}^{*}\left(x-T_{\alpha}(x-z)\right)\right| \leq 2 \alpha$, so Lemma 2.5 gives

$$
\begin{aligned}
\left\|P_{B \backslash A}\left(x-T_{\alpha}(x-z)\right)\right\| & \leq 2 \alpha \sup _{|\varepsilon|=1}\left\|\mathbf{1}_{\mathcal{\varepsilon}(B \backslash A)}\right\| \\
& \leq \frac{2}{t} \min _{n \in A \backslash B}\left|\mathbf{e}_{n}^{*}(x-z)\right| \sup _{|\mathcal{E}|=1}\left\|\mathbf{1}_{\mathcal{E}(B \backslash A)}\right\|=(*) .
\end{aligned}
$$

At this point we have two possible approaches. Let $\eta_{n}=\operatorname{sign}\left[e_{n}^{*}(x-z)\right]$. In the first approach we pick a greedy set $\Gamma \in G(x-z,|A \backslash B|, 1)$, and control (3.4) by

$$
(*) \leq \frac{2}{t} \min _{n \in \Gamma}\left|\mathbf{e}_{n}^{*}(x-z)\right| \tilde{\mu}_{m}\left\|\mathbf{1}_{\eta \Gamma}\right\| \leq \frac{2}{t} \tilde{\mu}_{m} \tilde{g}_{m}\|x-z\|,
$$

using Lemma 2.2 in the last step. In the second approach, we argue as follows

$$
(*) \leq \frac{2}{t} \min _{n \in A \backslash B}\left|\mathbf{e}_{n}^{*}(x-z)\right| \tilde{\mu}_{m}^{d}\left\|\mathbf{1}_{\eta(A \backslash B)}\right\| \leq \frac{2}{t} \gamma_{2 m} \tilde{g}_{2 m} \tilde{\mu}_{m}^{d}\|x-z\|
$$

using in the last step Lemma 2.3 and the fact that, if $\delta=\min _{A \backslash B}\left|\mathbf{e}_{n}^{*}(x-z)\right|$, then the set $(A \backslash B) \cup\left\{n:\left|\mathbf{e}_{n}^{*}(x-z)\right|>\delta\right\} \subset A \cup B$ and hence has cardinality $\leq 2 m$.

We can now combine the estimates displayed in (3.1)-(3.6) and obtain

$$
\left\|x-\mathfrak{C G}_{m}^{t} x\right\| \leq\left[g_{2 m}^{c}+\frac{2}{t} \min \left\{\tilde{g}_{m} \tilde{\mu}_{m}, \gamma_{2 m} \tilde{g}_{2 m} \tilde{\mu}_{m}^{d}\right\}\right]\|x-z\|,
$$

which after taking the infimum over all $z$ establishes Theorem 1.2 ,

Remark 3.1. In [18, Theorem 3.5] a stronger inequality is stated (for $t=1$ ), namely

$$
\mathbf{L}_{m}^{\mathrm{ch}} \leq g_{2 m}^{c}+2 \tilde{g}_{m} \tilde{\mu}_{m}^{d} .
$$

The proof, however, seems to contain a gap, and a missing factor $k_{m}^{c}$ should also appear in the last summand. Nevertheless, it is still fair to ask whether the inequality (3.7) asserted in [18] may be true with a different proof. 
Remark 3.2. Using Remark 2.4 in place of Lemma 2.3 in (3.6) above leads to an alternative and slightly simpler estimate

$$
\mathbf{L}_{m}^{\mathrm{ch}, t} \leq g_{2 m}^{c}+\frac{2}{t} k_{m} \tilde{\mu}_{m}^{d} .
$$

However, this would not be as efficient as (1.5) when $\mathscr{B}$ is quasi-greedy and conditional.

Remark 3.3. When $\mathscr{B}$ is quasi-greedy with constant $\mathbf{q}=\sup _{m} g_{m}<\infty$, then Theorem 1.2 implies the following

$$
\mathbf{L}_{m}^{\mathrm{ch}, t} \leq \mathbf{q}+4 t^{-1} \mathbf{q}^{2} \tilde{\mu}_{m}^{d} .
$$

This is a slight improvement with respect to [9, Theorem 4.1].

3.3. Proof of Theorem 1.3, Recall that $S_{N}=\sum_{n=1}^{N} \mathbf{e}_{n}^{*}(\cdot) \mathbf{e}_{n}$ and

$$
F_{N}(x)=\frac{1}{N} \sum_{n=1}^{N} S_{n}(x)=\sum_{n=1}^{N}\left(1-\frac{n-1}{N}\right) \mathbf{e}_{n}^{*}(x) \mathbf{e}_{n} .
$$

For $M>N$ we define the operators (of de la Vallée-Poussin type)

$$
\begin{aligned}
V_{N, M}(x) & =\frac{M}{M-N} F_{M}(x)-\frac{N}{M-N} F_{N}(x) \\
& =\sum_{n=1}^{N} \mathbf{e}_{n}^{*}(x) \mathbf{e}_{n}+\sum_{n=N+1}^{M}\left(1-\frac{n-N-1}{M-N}\right) \mathbf{e}_{n}^{*}(x) \mathbf{e}_{n} .
\end{aligned}
$$

In particular, observe that, for $\beta$ as in (1.1) we have

$$
\max \left\{\left\|V_{N, M}\right\|,\left\|I-V_{N, M}\right\|\right\} \leq \frac{M+N}{M-N} \beta .
$$

We next prove that, if $c \geq 2$, then for all $A, B \subset \mathbb{N}$ such that $B>c A$ with $|A|=|B| \leq m$ it holds

$$
\mathbf{L}_{m}^{\mathrm{ch}, t} \geq \frac{1}{t \beta} \frac{c-1}{c+1} \frac{\left\|\mathbf{1}_{\varepsilon A}\right\|}{\left\|\mathbf{1}_{\eta B}\right\|}, \quad \forall|\varepsilon|=|\eta|=1 .
$$

Pick any set $C>B$ such that $|B \cup C|=m$, and let

$$
x=\mathbf{1}_{\varepsilon A}+t \mathbf{1}_{\eta B}+t \mathbf{1}_{C} .
$$

Then $B \cup C \in G(x, m, t)$, and hence there is a Chebyshev $t$-greedy operator so that

$$
x-\mathfrak{C G}_{m}^{t}(x)=\mathbf{1}_{\varepsilon A}+\sum_{n \in B \cup C} a_{n} \mathbf{e}_{n},
$$

for some scalars $a_{n} \in \mathbb{K}$. Clearly,

$$
\left\|x-\mathfrak{C G}_{m}^{t}(x)\right\| \leq \mathbf{L}_{m}^{\mathrm{ch}, t} \sigma_{m}(x) \leq \mathbf{L}_{m}^{\mathrm{ch}, t}\left\|t \mathbf{1}_{\eta B}\right\|,
$$

using $z=\mathbf{1}_{\varepsilon A}+t \mathbf{1}_{C}$ an $m$-term approximant. On the other hand, let $N=\max A$. Since $\min B \cup C>c N$, then (3.9) yields

$$
V_{N, c N}\left(x-\mathfrak{C G}_{m}^{t} x\right)=\mathbf{1}_{\varepsilon A} .
$$

Therefore, (3.10) implies that

$$
\left\|x-\mathfrak{C G}_{m}^{t}(x)\right\| \geq \frac{\left\|V_{N, c N}\left(x-\mathfrak{C G}_{m}^{t} x\right)\right\|}{\left\|V_{N, c N}\right\|} \geq \frac{c-1}{(c+1) \beta}\left\|\mathbf{1}_{\varepsilon A}\right\| .
$$

We have therefore proved (3.11). 
We next show that when $|A|=|B| \leq m$ satisfy $A>c B$ then

$$
\mathbf{L}_{m}^{\mathrm{ch}, t} \geq \frac{1}{t \beta^{2}} \frac{c-1}{c+1} \frac{\left\|\mathbf{1}_{\varepsilon A}\right\|}{\left\|\mathbf{1}_{\eta B}\right\|}, \quad \forall|\varepsilon|=|\eta|=1
$$

This together with (3.11) is enough to establish Theorem 1.3. We shall actually show a slightly stronger result:

Lemma 3.4. Let $|A|=|B| \leq m$ and let $y \in \mathbb{X}$ be such that $|y|_{\infty}:=\sup _{n}\left|\mathbf{e}_{n}^{*}(y)\right| \leq 1$ and $A>c(B \cup \operatorname{supp} y)$. Then

$$
\mathbf{L}_{m}^{\mathrm{ch}, t} \geq \frac{1}{t \beta^{2}} \frac{c-1}{c+1} \frac{\left\|\mathbf{1}_{\varepsilon A}\right\|}{\left\|\mathbf{1}_{\eta B}+y\right\|}, \quad \forall|\varepsilon|=|\eta|=1 .
$$

Observe that the case $y=0$ in (3.13) yields (3.12). We now show (3.13). Pick a large integer $\lambda>1$ and a set $C>\lambda A$ such that $|B \cup C|=m$. Let

$$
x=\mathbf{1}_{\varepsilon A}+t y+t \mathbf{1}_{\eta B}+t \mathbf{1}_{C} .
$$

As before, $B \cup C \in G(x, m, t)$, and hence for some Chebyshev $t$-greedy operator we have

$$
x-\mathfrak{C G}_{m}^{t}(x)=\mathbf{1}_{\varepsilon A}+t y+\sum_{n \in B \cup C} a_{n} \mathbf{e}_{n},
$$

for suitable scalars $a_{n} \in \mathbb{K}$. Choosing $\mathbf{1}_{\varepsilon A}+t \mathbf{1}_{C}$ as $m$-term approximant of $x$ we see that

$$
\left\|x-\mathfrak{C G}_{m}^{t}(x)\right\| \leq \mathbf{L}_{m}^{\mathrm{ch}, t} \sigma_{m}(x) \leq \mathbf{L}_{m}^{\mathrm{ch}, t} t\left\|\mathbf{1}_{\eta B}+y\right\| .
$$

On the other hand, calling $N=\max (B \cup \operatorname{supp} y)$ and $L=\max A$ we have

$$
\left(I-V_{N, c N}\right) \circ V_{L, \lambda L}\left(x-\mathfrak{C G}_{m}^{t} x\right)=\mathbf{1}_{\varepsilon A}
$$

Thus,

$$
\left\|x-\mathfrak{C G}_{m}^{t}(x)\right\| \geq \frac{\left\|\mathbf{1}_{\varepsilon A}\right\|}{\left\|I-V_{N, c N}\right\|\left\|V_{L, \lambda L}\right\|} \geq \frac{c-1}{(c+1) \beta} \frac{\lambda-1}{(\lambda+1) \beta}\left\|\mathbf{1}_{\varepsilon A}\right\| .
$$

Therefore we obtain

$$
\mathbf{L}_{m}^{\mathrm{ch}, t} \geq \frac{1}{t \beta^{2}} \frac{c-1}{c+1} \frac{\lambda-1}{\lambda+1} \frac{\left\|\mathbf{1}_{\varepsilon A}\right\|}{\left\|\mathbf{1}_{\eta B}+y\right\|}
$$

which letting $\lambda \rightarrow \infty$ yields (3.13). This completes the proof of Lemma 3.4, and hence of Theorem 1.3 .

Remark 3.5. When $\mathscr{B}$ is a Schauder basis, a similar proof gives the following lower bound, which is also obtained in [18, Theorem 2.2]

$$
\mathbf{L}_{m}^{\mathrm{ch}, t} \geq \frac{1}{\left(K_{b}+1\right) t} \sup \left\{\frac{\left\|\mathbf{1}_{\varepsilon A}\right\|}{\left\|\mathbf{1}_{\eta B}\right\|}:|A|=|B|=m, A>B \text { or } B>A,|\varepsilon|=|\eta|=1\right\} .
$$

The statement for Cesàro bases, however, will be needed for the applications in $\$ 4.3$.

3.4. Lower bounds for general M-bases. Observe that

$$
\vartheta_{m, c}=\sup _{|A| \leq m} \vartheta_{c}(A), \quad \text { where } \quad \vartheta_{c}(A)=\sup _{\substack{B,|B|=|A| \\ B>c A \\ \varepsilon, \eta \in Y}} \max \left\{\frac{\left\|\mathbf{1}_{\varepsilon A}\right\|}{\left\|\mathbf{1}_{\eta B}\right\|}, \frac{\left\|\mathbf{1}_{\eta B}\right\|}{\left\|\mathbf{1}_{\varepsilon A}\right\|}\right\} .
$$

We consider a new parameter

$$
\vartheta_{m}=\sup _{|A| \leq m} \inf _{c \geq 1} \vartheta_{c}(A)
$$


We remark that, unlike $\vartheta_{m, c}$, the parameter $\vartheta_{m}$ depends on $\left\{\mathbf{e}_{n}\right\}_{n=1}^{\infty}$ but not on the reorderings of the system. We shall give a lower bound for $\mathbf{L}_{m}^{\mathrm{ch}, t}$ in terms of $\vartheta_{m}$ in a less restrictive situation than the Cesàro basis assumption on $\left\{\mathbf{e}_{n}\right\}_{n=1}^{\infty}$.

Given $\rho \geq 1$, we say that $\left\{\mathbf{e}_{n}\right\}_{n=1}^{\infty}$ is $\rho$-admissible if the following holds: for each finite set $A \subset \mathbb{N}$, there exists $n_{0}=n_{0}(A)$ such that, for all sets $B$ with $\min B \geq n_{0}$ and $|B| \leq|A|$,

$$
\left\|\sum_{n \in A} \alpha_{n} \mathbf{e}_{n}\right\| \leq \rho\left\|\sum_{n \in A \cup B} \alpha_{n} \mathbf{e}_{n}\right\|, \quad \forall \alpha_{n} \in \mathbb{K} .
$$

Observe that (3.15) implies that

$$
\left\|\sum_{n \in B} \alpha_{n} \mathbf{e}_{n}\right\| \leq(\rho+1)\left\|\sum_{n \in A \cup B} \alpha_{n} \mathbf{e}_{n}\right\|, \quad \forall \alpha_{n} \in \mathbb{K} .
$$

This condition is clearly satisfied by all Schauder and Cesàro bases (with $\rho=K_{b}$ or $\rho>\beta$ ), but we shall see below that it also holds in more general situations.

Proposition 3.6. Let $\left\{\mathbf{e}_{n}, \mathbf{e}_{n}^{*}\right\}_{n=1}^{\infty}$ be an M-basis such that $\left\{\mathbf{e}_{n}\right\}_{n=1}^{\infty}$ is $\rho$-admissible. Then

$$
\mathbf{L}_{m}^{\mathrm{ch}, t} \geq \frac{\vartheta_{m}}{(\rho+1) t}, \quad \forall m \in \mathbb{N}, \quad t \in(0,1]
$$

Proof. Fix $A \subset \mathbb{N}$ such that $|A| \leq m$. Choose $C$ disjoint with $A$ such that $|A \cup C|=m$. Let $n_{0}=n_{0}(A \cup C)$ as in the above definition, which we may assume larger than max $A \cup C$. Pick any $B$ with $\min B \geq n_{0}$ and $|B|=|A|$, and any $\varepsilon, \eta \in \Upsilon$. Let $x=t \mathbf{1}_{\varepsilon A}+t \mathbf{1}_{C}+\mathbf{1}_{\eta B}$. Then $A \cup C \in G(x, m, t)$, and there is a Chebyshev $t$-greedy operator with $\mathfrak{C G}_{m}^{t}(x)$ supported in $A \cup C$. Thus,

$$
\left\|x-\mathfrak{C G}_{m}^{t}(x)\right\| \leq \mathbf{L}_{m}^{\mathrm{ch}, t} \sigma_{m}(x) \leq \mathbf{L}_{m}^{\mathrm{ch}, t}\left\|x-\left(\mathbf{1}_{\eta B}+t \mathbf{1}_{C}\right)\right\|=\mathbf{L}_{m}^{\mathrm{ch}, t} t\left\|\mathbf{1}_{\varepsilon A}\right\| .
$$

On the other hand, using the property in (3.16) one obtains

$$
\left\|x-\mathfrak{C G}_{m}^{t}(x)\right\| \geq \frac{\left\|\mathbf{1}_{\eta B}\right\|}{\rho+1}
$$

Thus,

$$
\mathbf{L}_{m}^{\mathrm{ch}, t} \geq \frac{1}{(\rho+1) t} \frac{\left\|\mathbf{1}_{\eta B}\right\|}{\left\|\mathbf{1}_{\varepsilon A}\right\|}
$$

We now assume additionally that $\min B \geq n_{0}+m$, and pick $D \subset\left[n_{0}, n_{0}+m-1\right]$ such that $|B|+|D|=m$. Let $y=\mathbf{1}_{\varepsilon A}+t \mathbf{1}_{\eta B}+t \mathbf{1}_{D}$. Then $B \cup D \in G(y, m, t)$ and a similar reasoning gives

$$
\frac{\left\|\mathbf{1}_{\varepsilon A}\right\|}{\rho} \leq\left\|y-\mathfrak{C G}_{m}^{t}(y)\right\| \leq \mathbf{L}_{m}^{\mathrm{ch}, t} \sigma_{m}(y) \leq \mathbf{L}_{m}^{\mathrm{ch}, t} t\left\|\mathbf{1}_{\eta B}\right\|
$$

Thus,

$$
\mathbf{L}_{m}^{\mathrm{ch}, t} \geq \frac{1}{(\rho+1) t} \max \left\{\frac{\left\|\mathbf{1}_{\eta B}\right\|}{\left\|\mathbf{1}_{\varepsilon A}\right\|}, \frac{\left\|\mathbf{1}_{\varepsilon A}\right\|}{\left\|\mathbf{1}_{\eta B}\right\|}\right\}
$$

and taking the supremum over all $|B|=|A|$ with $B \geq\left(n_{0}+m\right) A$ and all $\varepsilon, \eta \in \Upsilon$, we see that

$$
\mathbf{L}_{m}^{\mathrm{ch}, t} \geq \frac{\vartheta_{n_{0}+m}(A)}{(\rho+1) t} \geq \frac{\inf _{c \geq 1} \vartheta_{c}(A)}{(\rho+1) t} .
$$

Finally, a supremum over all $|A| \leq m$ leads to (3.17). 
We now give some general conditions in $\left\{\mathbf{e}_{n}, \mathbf{e}_{n}^{*}\right\}_{n=1}^{\infty}$ and $\mathbb{X}$ under which $\rho$-admissibility holds. We recall a few standard definitions; see e.g. [12]. We use the notation $\left[\mathbf{e}_{n}\right]_{n \in A}=$ $\overline{\operatorname{span}}\left\{\mathbf{e}_{n}\right\}_{n \in A}$, for $A \subset \mathbb{N}$. A sequence $\left\{\mathbf{e}_{n}\right\}_{n=1}^{\infty}$ is weakly null if

$$
\lim _{n \rightarrow \infty} x^{*}\left(\mathbf{e}_{n}\right)=0, \quad \forall x^{*} \in \mathbb{X}^{*} .
$$

Given a subset $Y \subset \mathbb{X}^{*}$, we shall say that $\left\{\mathbf{e}_{n}\right\}_{n=1}^{\infty}$ is $Y$-null if

$$
\lim _{n \rightarrow \infty} y\left(\mathbf{e}_{n}\right)=0, \quad \forall y \in Y \text {. }
$$

Given $\kappa \in(0,1]$, we say that a set $Y \subset \mathbb{X}^{*}$ is $\kappa$-norming whenever

$$
\sup _{x^{*} \in Y,\left\|x^{*}\right\| \leq 1}\left|x^{*}(x)\right| \geq \kappa\|x\|, \quad \forall x \in \mathbb{X} .
$$

Proposition 3.7. Let $\left\{\mathbf{e}_{n}, \mathbf{e}_{n}^{*}\right\}_{n=1}^{\infty}$ be a biorthogonal system in $\mathbb{X} \times \mathbb{X}^{*}$. Suppose that the sequence $\left\{\tilde{\mathbf{e}}_{n}:=\left\|\mathbf{e}_{n}^{*}\right\| \mathbf{e}_{n}\right\}_{n=1}^{\infty} \subset \mathbb{X}$ is $Y$-null, for some subset $Y \subset \mathbb{X}^{*}$ which is $\kappa$-norming. Then $\left\{\mathbf{e}_{n}\right\}_{n=1}^{\infty}$ is $\rho$-admissible for every $\rho>1 / \kappa$.

Proof. Consider a finite set $A \subset \mathbb{N}$ with say $|A|=m$ and denote

$$
E:=\left[\mathbf{e}_{n}\right]_{n \in A} .
$$

Given $\varepsilon>0$, one can find a finite set $S \subset Y \cap\left\{x^{*} \in \mathbb{X}^{*}:\left\|x^{*}\right\|=1\right\}$ so that

$$
\max _{x^{*} \in S}\left|x^{*}(e)\right| \geq(1-\varepsilon) \kappa\|e\|, \quad \forall e \in E .
$$

Indeed, it suffices to verify the above inequality for $e$ of norm 1. Pick an $\varepsilon \kappa / 2$-net $\left(z_{k}\right)_{k=1}^{N}$ in the unit sphere of $E$. For any $k$ find a norm one $z_{k}^{*} \in Y$ so that $\left|z_{k}^{*}\left(z_{k}\right)\right|>(1-\varepsilon / 2) \kappa$. We claim that $S=\left\{z_{k}^{*}: 1 \leq k \leq N\right\}$ has the desired properties. To see this, pick a norm one $e \in E$, and find $k$ with $\left\|e-z_{k}\right\| \leq \varepsilon \kappa / 2$. Then

$$
\max _{x^{*} \in S}\left|x^{*}(e)\right| \geq\left|z_{k}^{*}(e)\right| \geq\left|z_{k}^{*}\left(z_{k}\right)\right|-\left\|e-z_{k}\right\| \geq(1-\varepsilon / 2) \kappa-\varepsilon \kappa / 2=(1-\varepsilon) \kappa .
$$

Next, since the sequence $\left\{\left\|\mathbf{e}_{n}^{*}\right\| \mathbf{e}_{n}\right\}$ is $Y$-null, for each $\delta>0$ we can find an integer $n_{0}>$ $\max A$ so that

$$
\max _{x^{*} \in S}\left|x^{*}\left(\mathbf{e}_{n}\right)\right|\left\|\mathbf{e}_{n}^{*}\right\| \leq \frac{\delta \kappa}{m}, \quad \forall n \geq n_{0} .
$$

Pick any $B$ of cardinality $m$ with $\min B \geq n_{0}$, and let

$$
G:=\left[\mathbf{e}_{n}\right]_{n \in B} .
$$

For $f=\sum_{n \in B} \mathbf{e}_{n}^{*}(f) \mathbf{e}_{n} \in G$, we have

$$
\max _{x^{*} \in S}\left|x^{*}(f)\right| \leq \max _{x^{*} \in S} \sum_{n \in B}\left|x^{*}\left(\mathbf{e}_{n}\right)\right|\left\|\mathbf{e}_{n}^{*}\right\|\|f\| \leq \delta \kappa\|f\| .
$$

We claim that

$$
\|e+f\| \geq \frac{(1-\varepsilon-\delta) \kappa}{1+\delta \kappa}\|e\|, \text { for any } e \in E, f \in G
$$

To show this, we fix $\gamma>0$ (to be chosen later), and assume first that $\|f\| \geq(1+\gamma)\|e\|$. Then,

$$
\|e+f\| \geq\|f\|-\|e\| \geq \gamma\|e\| \text {. }
$$

Next assume that $\|f\|<(1+\gamma)\|e\|$, then using (3.18) and (3.19) we obtain that

$$
\|e+f\| \geq \max _{x^{*} \in S}\left|x^{*}(e+f)\right| \geq(1-\varepsilon) \kappa\|e\|-\delta \kappa\|f\|>(1-\varepsilon-\delta(1+\gamma)) \kappa\|e\| .
$$


We now choose $\gamma$ so that $\gamma=(1-\varepsilon-\delta(1+\gamma)) \kappa$, that is,

$$
\gamma=\frac{(1-\varepsilon-\delta) \kappa}{1+\delta \kappa}
$$

which shows the claim in (3.20). Now, given $\rho>1 / \kappa$, we may pick $\delta=\varepsilon$ sufficiently small so that the above number $\gamma>1 / \rho$. Then, (3.20) becomes

$$
\|e+f\| \geq \frac{1}{\rho}\|e\|, \text { for any } e \in\left[e_{n}\right]_{n \in A}, f \in\left[e_{n}\right]_{n \in B},
$$

for all $B$ with $\min B \geq n_{0}$ and $|B|=|A|=m$. Thus, $\left\{\mathbf{e}_{n}\right\}_{n=1}^{\infty}$ is $\rho$-admissible.

We mention a few cases where the hypotheses in the above proposition can be applied:

(1) When the sequence $\left\{\tilde{\mathbf{e}}_{n}\right\}_{n=1}^{\infty}$ is weakly null, since $Y=\mathbb{X}^{*}$ is always 1-norming.

(2) When $\sup _{n>1}\left\|\mathbf{e}_{n}\right\|\left\|\mathbf{e}_{n}^{*}\right\|<\infty$ and $Y=\left[\mathbf{e}_{n}^{*}\right]_{n \in \mathbb{N}}$ is $\kappa$-norming, since the first condition implies that $\left\{\tilde{\mathbf{e}}_{n}\right\}_{n=1}^{\infty}$ is $Y$-null. In particular, when $\left\{\mathbf{e}_{n}\right\}_{n=1}^{\infty}$ is a Schauder basis in $\mathbb{X}$, in which case the above conditions hold with $\kappa=1 / K_{b}$; see [19, Theorems I.3.1 and I.12.2].

(3) In every separable Banach space $\mathbb{X}$, if one picks $\left\{\mathbf{e}_{n}, \mathbf{e}_{n}^{*}\right\}_{n=1}^{\infty}$ to be an $M$-basis with the properties in (2) and $\kappa=1$; see e.g. [20, Theorem III.8.5] for the existence of such bases.

(4) Let $\mathbb{X}=C(K)$ where $K$ is a compact Hausdorff set and let $\mu$ be a Radon probability measure in $K$ with $\operatorname{supp} \mu=K$. Then, the natural embedding of $C(K)$ into $L_{\infty}(\mu)$ is isometric, and therefore $Y=L_{1}(\mu)$ is 1-norming in $\mathbb{X}$. Let $\left\{\mathbf{e}_{n}\right\}_{n=1}^{\infty}$ be a complete system in $\mathbb{X}$ which is orthonormal with respect to $\mu$ and uniformly bounded, that is, $\int_{K} \mathbf{e}_{n} \overline{\mathbf{e}_{m}} d \mu=\delta_{n, m}$ and $\sup _{n}\left\|\mathbf{e}_{n}\right\|_{\infty}<\infty$. Then the sequence $\left\{\mathbf{e}_{n}\right\}_{n=1}^{\infty}$ is $L_{1}(\mu)$-null in $\mathbb{X}$. Indeed, this follows from case (2), and the fact that $C(K)$ is dense in $L_{1}(\mu)$.

Examples of such systems in $C(K)$ include the trigonometric system in $C[0,1]$ (in the real or complex case), as well as certain polygonal versions of the Walsh system [6, 16, 26], or any reorderings of them (which may cease to be Cesàro bases).

(5) As a dual of the previous, if $\mathbb{X}=L^{1}(\mu)$ then every system $\left\{\mathbf{e}_{n}\right\}_{n=1}^{\infty}$ as in (4) is weakly null, and hence case (1) applies.

(6) Recall the definition of the right fundamental function: $\varphi_{r}(m)=\sup \left\{\left\|\mathbf{1}_{A}\right\|:|A| \leq m\right\}$. If $\left\{\mathbf{e}_{n}\right\}_{n=1}^{\infty}$ is such that $\varphi_{r}(m)=\mathbf{o}(m)$, then this system is weakly null. Indeed, first note that also $\tilde{\varphi}_{r}(m)=\sup \left\{\left\|\mathbf{1}_{\eta A}\right\|:|A| \leq m,|\eta|=1\right\}=\mathbf{o}(m)$. Assume that the system is not weakly null. Then there exist a norm one $x^{*} \in \mathbb{X}^{*}$ and $\varepsilon_{0}>0$ so that the set $A=\left\{n \in \mathbb{N}:\left|x^{*}\left(\mathbf{e}_{n}\right)\right| \geq\right.$ $\left.\varepsilon_{0}\right\}$ is infinite. Pick any $F \subset A$ with $|F|=m$ and let $\eta_{n}=\operatorname{sign}\left[x^{*}\left(\mathbf{e}_{n}\right)\right]$; then

$$
\tilde{\varphi}_{r}(m) \geq\left\|\mathbf{1}_{\bar{\eta} F}\right\| \geq\left|x^{*}\left(\sum_{n \in F} \overline{\eta_{n}} \mathbf{e}_{n}\right)\right|=\sum_{n \in F}\left|x^{*}\left(\mathbf{e}_{n}\right)\right| \geq m \varepsilon_{0}
$$

contradicting our assumption.

Finally, as a consequence of Propositions 3.6 and 3.7 one obtains

Theorem 3.8. Let $\left\{\mathbf{e}_{n}, \mathbf{e}_{n}^{*}\right\}_{n=1}^{\infty}$ be a seminormalized M-basis such that the sequence $\left\{\mathbf{e}_{n}\right\}_{n=1}^{\infty}$ is $Y$-null for some subset $Y \subset \mathbb{X}^{*}$ which is $\kappa$-norming. Then, if $\vartheta_{m}$ is as in (3.14), we have

$$
\mathbf{L}_{m}^{\mathrm{ch}, t} \geq \frac{\kappa \vartheta_{m}}{(\kappa+1) t}, \quad \forall m \in \mathbb{N}, \quad t \in(0,1]
$$




\section{EXAMPLES}

The first two examples are variants of those in [4, §5.1] and [5, §8.1].

4.1. Example 4.1; The summing basis. Let $\mathbb{X}$ be the closure of the set of all finite sequences $\mathbf{a}=\left(a_{n}\right)_{n} \in c_{00}$ with the norm

$$
\|\mathbf{a}\|=\sup _{m}\left|\sum_{n=1}^{m} a_{n}\right|
$$

The canonical system $\mathscr{B}=\left\{\mathbf{e}_{n}\right\}_{n=1}^{\infty}$ is a Schauder basis in $\mathbb{X}$ with $K_{b}=1$ and $\left\|\mathbf{e}_{n}\right\|=1$ for all $n$. Also, $\left\|\mathbf{e}_{1}^{*}\right\|=1,\left\|\mathbf{e}_{n}^{*}\right\|=2$ if $n \geq 2$, so $\mathfrak{K}=2$ in Theorem 1.1, see [4, §5.1]. We now show that, for this example of $(\mathbb{X}, \mathscr{B})$, the bound of Theorem 1.1 is sharp. As in [4, §5.1], we consider the element:

$$
x=(\underbrace{\frac{1}{2}, \frac{1}{t}, \frac{1}{2}}, \ldots, \underbrace{\frac{1}{2}, \frac{1}{t}, \frac{1}{2}} ; \frac{1}{2} ; \underbrace{-1,1}, \ldots, \underbrace{-1,1}, 0, \ldots),
$$

where we have $m$ blocks of $\left(\frac{1}{2}, \frac{1}{t}, \frac{1}{2}\right)$ and $m$ blocks of $(-1,1)$. Picking $A=\left\{n: x_{n}=-1\right\}$ as a $t$-greedy set of $x$, we see that

$$
\begin{aligned}
\left\|x-\mathfrak{C G G}_{m}^{t}(x)\right\| & =\min _{a_{i}, i=1, \ldots, m}\left\|\left(\frac{1}{2}, \frac{1}{t}, \frac{1}{2}, \ldots, \frac{1}{2}, \frac{1}{t}, \frac{1}{2} ; \frac{1}{2} ; a_{1}, 1, a_{2}, 1, \ldots, a_{m}, 1,0, \ldots\right)\right\| \\
& \geq\left\|\left(\frac{1}{2}, \frac{1}{t}, \frac{1}{2}, \ldots, \frac{1}{2}, \frac{1}{t}, \frac{1}{2} ; \frac{1}{2} ; 0, \ldots\right)\right\|=m+\frac{m}{t}+\frac{1}{2} .
\end{aligned}
$$

On the other hand,

$$
\begin{aligned}
\sigma_{m}(x) & \leq\left\|x-\frac{t+1}{t}(0,1,0, \ldots, 0,1,0 ; 0, \ldots)\right\| \\
& =\left\|\left(\frac{1}{2},-1, \frac{1}{2}, \ldots, \frac{1}{2},-1, \frac{1}{2} ; \frac{1}{2} ;-1,1, \ldots,-1,1,0 \ldots\right)\right\|=\frac{1}{2} .
\end{aligned}
$$

Hence, $\mathbf{L}_{m}^{\mathrm{ch}, t} \geq 1+2\left(1+\frac{1}{t}\right) m$ and we conclude that $\mathbf{L}_{m}^{\mathrm{ch}, t}=1+2\left(1+\frac{1}{t}\right) m$ by Theorem 1.1 . As a consequence, observe that in this case $\mathfrak{C G}_{m}^{t}(x)=0$.

Remark 4.1. The above example strengthens [18, Theorem 2.4], where the authors are only able to show that $1+4 m \leq \mathbf{L}_{m}^{\text {ch }} \leq 1+6 m$.

4.2. Example 4.2: the difference basis. Let $\left\{\mathbf{e}_{n}\right\}_{n=1}^{\infty}$ be the canonical basis in $\ell^{1}(\mathbb{N})$ and define the elements

$$
y_{1}=\mathbf{e}_{1}, y_{n}=\mathbf{e}_{n}-\mathbf{e}_{n-1}, n=2,3, \ldots
$$

The new system $\mathscr{B}=\left\{y_{n}\right\}_{n=1}^{\infty}$ is called the difference basis of $\ell^{1}$. We recall some basic properties used in [5, §8.1]. If $\left(b_{n}\right)_{n} \in c_{00}$ then

$$
\left\|\sum_{n=1}^{\infty} b_{n} y_{n}\right\|=\sum_{n=1}^{\infty}\left|b_{n}-b_{n+1}\right| \text {. }
$$

Also, $\mathscr{B}$ is a monotone basis with $\left\|y_{1}\right\|=1,\left\|y_{n}\right\|=2$ if $n \geq 2$, and $\left\|y_{n}^{*}\right\|=1$ for all $n \geq 1$ (in fact, the dual system corresponds to the summing basis). So, $\mathfrak{K}=2$ and Theorem 1.1 gives $\mathbf{L}_{m}^{\mathrm{ch}, t} \leq 1+2\left(1+\frac{1}{t}\right) m$ for all $t \in(0,1]$. To show the equality we consider the vector $x=\sum_{n} b_{n} y_{n}$ with coefficients $\left(b_{n}\right)$ given by

$$
(1, \underbrace{1,1,-\frac{1}{t}, 1}, \ldots, \underbrace{1,1,-\frac{1}{t}, 1}, 0, \ldots)
$$


where the block $\left(1,1, \frac{-1}{t}, 1\right)$ is repeated $m$ times. If we take $\Gamma=\{2,6, \ldots, 4 m-2\}$ as a $t$-greedy set for $x$ of cardinality $m$, then

$$
\begin{aligned}
\left\|x-\mathfrak{C G}_{m}^{t}(x)\right\| & =\inf _{\left(a_{j}\right)_{j=1}^{m}}\left\|x-\sum_{j=1}^{m} a_{j} y_{4 j-2}\right\| \\
& =\inf _{\left(a_{j}\right)_{j=1}^{m}}\left\|\left(1,1-a_{1}, 1, \frac{-1}{t}, 1, \ldots, 1-a_{m}, 1, \frac{-1}{t}, 1,0, \ldots\right)\right\| \\
& =\inf _{\left(a_{j}\right)_{j=1}^{m}} 2 \sum_{j=1}^{m}\left|a_{j}\right|+2 m\left(1+\frac{1}{t}\right)+1=2 m\left(1+\frac{1}{t}\right)+1 .
\end{aligned}
$$

Hence, in this case we also have $\mathfrak{C G}_{m}^{t}(x)=0$. On the other hand

$$
\sigma_{m}(x) \leq\left\|x+\left(1+\frac{1}{t}\right) \sum_{j=1}^{m} y_{4 j}\right\|=\|(1,1,1,1,1, \ldots, 1,1,1,1,0, \ldots)\|=1 .
$$

This shows that $\mathbf{L}_{m}^{\mathrm{ch}, t}=1+2\left(1+\frac{1}{t}\right) m$.

4.3. Example 4.3; the trigonometric system in $L^{p}(\mathbb{T})$. Consider $\mathscr{B}=\left\{e^{i n x}\right\}_{n \in \mathbb{Z}}$ in $L^{p}(\mathbb{T})$ for $1 \leq p<\infty$, and in $C(\mathbb{T})$ if $p=\infty$. In [21], Temlyakov showed that

$$
c_{p} m^{\left|\frac{1}{p}-\frac{1}{2}\right|} \leq \mathbf{L}_{m} \leq 1+3 m^{\left|\frac{1}{p}-\frac{1}{2}\right|},
$$

for some $c_{p}>0$ and all $1 \leq p \leq \infty$. Adapting his argument, Shao and Ye have recently established, in [18, Theorem 2.1], that for $1<p \leq \infty$ it also holds

$$
\mathbf{L}_{m}^{\mathrm{ch}} \approx m^{\left|\frac{1}{p}-\frac{1}{2}\right|} .
$$

The case $p=1$ is left as an open question, and only the estimate $\frac{\sqrt{m}}{\ln (m)} \lesssim \mathbf{L}_{m}^{\mathrm{ch}} \lesssim \sqrt{m}$ is given; see [18, (2.24)]. Moreover, the proof of the case $p=\infty$ seems to contain some gaps and may not be complete.

Here, we shall give a short proof ensuring the validity of (4.1) in the full range $1 \leq p \leq \infty$, with a reasoning similar to [4, §5.4]. More precisely, we shall prove the following.

Proposition 4.2. Let $1 \leq p \leq \infty$. Then there exists $c_{p}>0$ such that

$$
\mathbf{L}_{m}^{\mathrm{ch}, t} \geq c_{p} t^{-1} m^{\left|\frac{1}{p}-\frac{1}{2}\right|}, \quad \forall m \in \mathbb{N}, \quad t \in(0,1] .
$$

We remark that in the cases $p=1$ and $p=\infty$ the trigonometric system is not a Schauder basis, but it is a Cesàro basis 1 . So we may use the lower bounds in Theorem 1.3 , namely

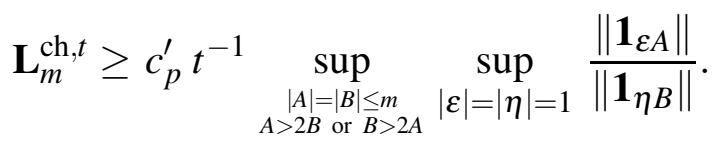

- Case $1<p \leq 2$. Assume that $m=2 \ell+1$ or $2 \ell+2$ (that is, $\ell=\left\lfloor\frac{m-1}{2}\right\rfloor$ ). We choose $B=\{-\ell, \ldots, \ell\}$, so that $\mathbf{1}_{B}=D_{\ell}$ is the $\ell$-th Dirichlet kernel, and hence

$$
\left\|\mathbf{1}_{B}\right\|_{p}=\left\|D_{\ell}\right\|_{L^{p}(\mathbb{T})} \approx m^{1-\frac{1}{p}} .
$$

Next we take a lacunary set $A=\left\{2^{j}: j_{0} \leq j \leq j_{0}+2 \ell\right\}$, so that

$$
\left\|\mathbf{1}_{A}\right\|_{p} \approx \sqrt{m}
$$

\footnotetext{
${ }^{1}$ We equip $\mathscr{B}$ with its natural ordering $\left\{1, e^{i x}, e^{-i x}, e^{2 i x}, e^{-2 i x}, \ldots\right\}$.
} 
and where $j_{0}$ is chosen such that $2^{j_{0}} \geq m$, and hence $A>2 B$. Then, (4.3) implies

$$
\mathbf{L}_{m}^{\mathrm{ch}, t} \geq c_{p} t^{-1} \frac{m^{1 / 2}}{m^{1-\frac{1}{p}}}=c_{p} t^{-1} m^{\left|\frac{1}{p}-\frac{1}{2}\right|} .
$$

- Case $2 \leq p<\infty$. The same proof works in this case, just reversing the roles of $A$ and $B$.

- Case $p=\infty$. We replace the lacunary set by a Rudin-Shapiro polynomial of the form

$$
R(x)=e^{i N x} \sum_{n=0}^{2^{L}-1} \varepsilon_{n} e^{i n x}, \quad \text { with } \varepsilon_{n} \in\{ \pm 1\}
$$

where $L$ is such that $2^{L} \leq m<2^{L+1}$; see e.g. [13, p. 33]. Then, $R=\mathbf{1}_{\varepsilon B}$ with $B=N+\left\{0,1, \ldots, 2^{L}-1\right\}$ and

$$
\left\|\mathbf{1}_{\varepsilon B}\right\|_{\infty}=\|R\|_{L^{\infty}(\mathbb{T})} \approx \sqrt{m} .
$$

If we pick $N \geq 2 \cdot 2^{L}$, then $B>2 A$ with $A=\left\{ \pm 1, \ldots, \pm\left(2^{L}-1\right)\right\}$. Finally,

$$
\left\|\mathbf{1}_{A}\right\|_{\infty}=\left\|D_{2^{L}-1}-1\right\|_{L^{\infty}(\mathbb{T})} \approx m
$$

So, (4.3) implies the desired bound.

- Case $p=1$. We use the lower bound in Lemma 3.4 namely

$$
\mathbf{L}_{m}^{\mathrm{ch}, t} \geq c_{1}^{\prime} t^{-1} \frac{\left\|\mathbf{1}_{A}\right\|}{\left\|\mathbf{1}_{B}+y\right\|}
$$

for all $|A|=|B| \leq m$ and all $y$ such that $A>2(B \cup \operatorname{supp} y)$ and $\sup _{n}\left|\mathbf{e}_{n}^{*}(y)\right| \leq 1$. As before, let $m=2 \ell+1$ or $2 \ell+2$, and choose the same sets $A$ and $B$ as in the case $1<p \leq 2$. Next choose $y$ so that the vector

$$
V_{\ell}=\mathbf{1}_{B}+y
$$

is a de la Vallée-Poussin kernel as in [13, p. 15]. Then, the Fourier coeffients $\mathbf{e}_{n}^{*}(y)$ have modulus $\leq 1$ and are supported in $\{n: \ell<|n| \leq 2 \ell+1\}$, so the condition $A>2(B \cup \operatorname{supp} y)$ holds if $2^{j_{0}} \geq 2 m+1$. Finally,

$$
\left\|\mathbf{1}_{B}+y\right\|_{1}=\left\|V_{\ell}\right\|_{L^{1}(\mathbb{T})} \leq 3
$$

so the bound $\mathbf{L}_{m}^{\mathrm{ch}, t} \gtrsim t^{-1} \sqrt{m}$ follows from (4.5).

Remark 4.3. Using the trivial upper bound $\mathbf{L}_{m}^{\mathrm{ch}, t} \leq \mathbf{L}_{m}^{t} \lesssim t^{-1} m^{\left|\frac{1}{p}-\frac{1}{2}\right|}$, we conclude that $\mathbf{L}_{m}^{\mathrm{ch}, t} \approx t^{-1} m^{\left|\frac{1}{p}-\frac{1}{2}\right|}$ for all $1 \leq p \leq \infty$.

\section{COMPARISON BETWEEN $\tilde{\mu}_{m}$ AND $\tilde{\mu}_{m}^{d}$}

In this section we compare the democracy constants $\tilde{\mu}_{m}$ and $\tilde{\mu}_{m}^{d}$ defined in $\S 1$ above. Let us first note that

$$
\tilde{\mu}_{m}^{d} \leq \tilde{\mu}_{m} \leq\left(\tilde{\mu}_{m}^{d}\right)^{2}
$$

and

$$
\tilde{\mu}_{m}^{d} \leq \tilde{\mu}_{m} \leq(1+2 \kappa) \gamma_{m} \tilde{\mu}_{m}^{d}
$$


where $\kappa=1$ or 2 depending if $\mathbb{K}=\mathbb{R}$ or $\mathbb{C}$. Indeed, the left inequality in (5.1) is immediate by definition, and the right one follows from

$$
\frac{\left\|\mathbf{1}_{\eta B}\right\|}{\left\|\mathbf{1}_{\varepsilon A}\right\|}=\frac{\left\|\mathbf{1}_{\eta B}\right\|}{\left\|\mathbf{1}_{C}\right\|} \frac{\left\|\mathbf{1}_{C}\right\|}{\left\|\mathbf{1}_{\varepsilon A}\right\|} \leq\left(\tilde{\mu}_{m}^{d}\right)^{2}
$$

for any $|A|=|B| \leq m$ and any $C$ disjoint with $A \cup B$ with $|C|=|A|=|B|$. Concerning the right inequality in (5.2), we use that if $|A|=|B| \leq m$ then

$$
\frac{\left\|\mathbf{1}_{\varepsilon A}\right\|}{\left\|\mathbf{1}_{\eta B}\right\|} \leq \frac{\left\|\mathbf{1}_{\varepsilon(A \backslash B)}\right\|+\left\|\mathbf{1}_{\varepsilon(A \cap B)}\right\|}{\left\|\mathbf{1}_{\eta B}\right\|} \leq \gamma_{m} \frac{\left\|\mathbf{1}_{\varepsilon(A \backslash B)}\right\|}{\left\|\mathbf{1}_{\eta(B \backslash A)}\right\|}+\frac{\left\|\mathbf{1}_{\varepsilon(A \cap B)}\right\|}{\left\|\mathbf{1}_{\eta B}\right\|} \leq \gamma_{m} \tilde{\mu}_{m}^{d}+2 \kappa \gamma_{m},
$$

using in the last step [4, Lemma 3.3]. From (5.2) we see that $\tilde{\mu}_{m} \approx \tilde{\mu}_{m}^{d}$ when $\mathscr{B}$ is quasigreedy for constant coefficients.

In the next subsection we shall show that $\tilde{\mu}_{m} \approx \tilde{\mu}_{m}^{d}$ for all Schauder bases, a result which seems new in the literature.

5.1. Equivalence for Schauder bases. We begin with a simple observation.

\section{Lemma 5.1.}

$$
\tilde{\mu}_{m}^{d}=\sup \left\{\frac{\left\|\mathbf{1}_{\eta B}\right\|}{\left\|\mathbf{1}_{\varepsilon A}\right\|}:|B| \leq|A| \leq m, A \cap B=\emptyset,|\varepsilon|=|\eta|=1\right\} .
$$

Proof. Let $|\varepsilon|=|\eta|=1$ and $|B| \leq|A| \leq m$ with $A \cap B=\emptyset$. We must show that $\left\|\mathbf{1}_{\eta B}\right\| /\left\|\mathbf{1}_{\varepsilon A}\right\| \leq$ $\tilde{\mu}_{m}^{d}$. Pick any set $C$ disjoint with $A \cup B$ such that $|B|+|C|=|A|$. We now use the elementary inequality

$$
\|x\|=\left\|\frac{x+y}{2}+\frac{x-y}{2}\right\| \leq \max \{\|x+y\|,\|x-y\|\},
$$

with $x=\mathbf{1}_{\eta B}$ and $y=\mathbf{1}_{C}$. Let $\eta^{\prime} \in \Upsilon$ be such that $\left.\eta^{\prime}\right|_{B}=\left.\eta\right|_{B}$ and $\left.\eta^{\prime}\right|_{C}= \pm 1$, according to the sign that reaches the maximum in (5.4). Then $\left\|\mathbf{1}_{\eta B}\right\| \leq\left\|\mathbf{1}_{\eta^{\prime}(B \cup C)}\right\| \leq \tilde{\mu}_{m}^{d}\left\|\mathbf{1}_{\varepsilon A}\right\|$, and the result follows.

Theorem 5.2. If $K_{b}$ is the basis constant and $\varkappa=\sup _{n}\left\|\mathbf{e}_{n}^{*}\right\|\left\|\mathbf{e}_{n}\right\|$, then

$$
\tilde{\mu}_{m} \leq 2\left(K_{b}+1\right) \tilde{\mu}_{m}^{d}+\varkappa K_{b} .
$$

Proof. Let $|A|=|B| \leq m$, and $|\varepsilon|=|\eta|=1$. Then

$$
\frac{\left\|\mathbf{1}_{\eta B}\right\|}{\left\|\mathbf{1}_{\varepsilon A}\right\|} \leq \frac{\left\|\mathbf{1}_{\eta(B \backslash A)}\right\|}{\left\|\mathbf{1}_{\varepsilon A}\right\|}+\frac{\left\|\mathbf{1}_{\eta(B \cap A)}\right\|}{\left\|\mathbf{1}_{\varepsilon A}\right\|}=I+I I .
$$

Lemma 5.1 implies $I \leq \tilde{\mu}_{m}^{d}$. We now bound $I I$. Pick an integer $n_{0}$ such that $A_{1}=\{n \in$ $\left.A: n \leq n_{0}\right\}$ and $A_{2}=A \backslash A_{1}$ satisfy

$$
\left|A_{1}\right|=\left|A_{2}\right| \quad \text { (if }|A| \text { is even), or } \quad\left|A_{1}\right|=\frac{|A|-1}{2}=\left|A_{2}\right|-1 \quad \text { (if }|A| \text { is odd). }
$$

Then

$$
\begin{aligned}
I I & \leq \frac{\left\|\mathbf{1}_{\eta\left(B \cap A_{1}\right)}\right\|}{\left\|\mathbf{1}_{\varepsilon A}\right\|}+\frac{\left\|\mathbf{1}_{\eta\left(B \cap A_{2}\right)}\right\|}{\left\|\mathbf{1}_{\varepsilon A}\right\|} \\
& \leq\left(K_{b}+1\right) \frac{\left\|\mathbf{1}_{\eta\left(B \cap A_{1}\right)}\right\|}{\left\|\mathbf{1}_{\varepsilon A_{2}}\right\|}+K_{b} \frac{\left\|\mathbf{1}_{\eta\left(B \cap A_{2}\right)}\right\|}{\left\|\mathbf{1}_{\varepsilon A_{1}}\right\|}=I I_{1}+I I_{2}
\end{aligned}
$$


using in the second line the basis constant bound for the denominator. Since $\left|B \cap A_{1}\right| \leq$ $\left|A_{1}\right| \leq\left|A_{2}\right|$, we see that

$$
I_{1} \leq\left(K_{b}+1\right) \tilde{\mu}_{m}^{d} .
$$

On the other hand, picking any number $n_{1} \in B \cap A_{2}$, and using $\left\|\mathbf{e}_{n_{1}}^{*}\right\|\left\|\mathbf{1}_{\varepsilon A}\right\| \geq\left|\mathbf{e}_{n_{1}}^{*}\left(\mathbf{1}_{\varepsilon A}\right)\right|=1$, we see that

$$
I I_{2} \leq K_{b} \frac{\left\|\mathbf{1}_{\eta\left(B \cap A_{2} \backslash\left\{n_{1}\right\}\right)}\right\|}{\left\|\mathbf{1}_{\varepsilon A_{1}}\right\|}+K_{b}\left\|\mathbf{e}_{n_{1}}\right\|\left\|\mathbf{e}_{n_{1}}^{*}\right\| \leq K_{b} \tilde{\mu}_{m}^{d}+\varkappa K_{b},
$$

the last bound due to $\left|B \cap A_{2} \backslash\left\{n_{1}\right\}\right| \leq\left|A_{2}\right|-1 \leq\left|A_{1}\right|$ and Lemma 5.1. Putting together the previous bounds easily leads to (5.5).

Remark 5.3. A similar argument shows the equivalence of the standard (unsigned) democracy parameters

$$
\mu_{m}=\sup _{|A|=|B| \leq m} \frac{\left\|\mathbf{1}_{B}\right\|}{\left\|\mathbf{1}_{A}\right\|} \quad \text { and } \quad \mu_{m}^{d}=\sup _{\substack{|A|=|B| \leq m \\ A \cap B=\emptyset}} \frac{\left\|\mathbf{1}_{B}\right\|}{\left\|\mathbf{1}_{A}\right\|} .
$$

Indeed, in this case, the analog of (5.3) takes the weaker form

$$
\mu_{m}^{d} \leq \sup _{\substack{|B| \leq|A| \leq m \\ A \cap B=\emptyset}} \frac{\left\|\mathbf{1}_{B}\right\|}{\left\|\mathbf{1}_{A}\right\|} \leq K_{b} \mu_{m}^{d}
$$

Then, (5.7) and the same proof we gave for Theorem 5.2 (with $\eta=\varepsilon \equiv 1$ ) leads to

$$
\mu_{m} \leq 2\left(K_{b}+1\right) K_{b} \mu_{m}^{d}+\varkappa K_{b} .
$$

5.2. An example where $\tilde{\mu}_{m}$ grows faster than $\tilde{\mu}_{m}^{d}$. The following example also seems to be new in the literature. As in (5.6), we denote by $\mu_{m}, \mu_{m}^{d}$ the democracy parameters corresponding to constant signs.

Theorem 5.4. There exists a Banach space $\mathbb{X}$ with an $M$-basis $\mathscr{B}$ such that

$$
\limsup _{m \rightarrow \infty} \frac{\tilde{\mu}_{m}}{\left[\tilde{\mu}_{m}^{d}\right]^{2-\varepsilon}}=\limsup _{m \rightarrow \infty} \frac{\mu_{m}}{\left[\mu_{m}^{d}\right]^{2-\varepsilon}}=\infty, \quad \forall \varepsilon>0 .
$$

Proof. Let $N_{0}=1$, and define recursively $N_{k}=2^{2^{N_{k-1}}}$, and $N_{k}^{\prime}=N_{1}+\ldots+N_{k-1}$. Consider the blocks of integers

$$
S_{k}=\left\{N_{k}^{\prime}+1, \ldots, N_{k}^{\prime}+N_{k}\right\},
$$

and denote the tail blocks by $T_{k}=\cup_{j \geq k+1} S_{j}$. Finally, let

$$
\mathfrak{N}_{k}=\left\{\left(\sigma_{j}\right)_{j \in S_{k}}: \sigma_{j} \in\{ \pm 1\} \quad \text { and } \quad \sum_{j \in S_{k}} \sigma_{j}=0\right\}
$$

We define a real Banach space $\mathbb{X}$ as the closure of $c_{00}$ with the norm

$$
\|x\|=\max \left\{\|x\|_{\infty}, \sup _{k \geq 1} \alpha_{k} \sup _{\sigma \in \mathfrak{N}_{k}}\left|\left\langle\mathbf{1}_{\sigma S_{k}}, x\right\rangle\right|, \sup _{k \geq 1} \beta_{k} \sup _{\substack{S \subset T_{k} \\|S|=N_{k}}} \sum_{j \in S}\left|x_{j}\right|\right\},
$$

where the weights $\alpha_{k}$ and $\beta_{k}$ are chosen as follows:

$$
\alpha_{k}=2^{-N_{k-1}}=\frac{1}{\log _{2} N_{k}} \quad \text { and } \quad \beta_{k}=\frac{1}{\sqrt{N_{k}}} .
$$


Observe that

$$
N_{k}^{\prime}=N_{1}+\ldots+N_{k-1} \leq 2 N_{k-1}=2 \log _{2} \log _{2} N_{k} \quad \text { and } \quad \frac{\alpha_{k}}{\beta_{k}}=\frac{\sqrt{N_{k}}}{\log _{2} N_{k}} .
$$

Claim 1: $\quad \tilde{\mu}_{N_{k}} \geq \mu_{N_{k}} \geq \frac{N_{k} / 2}{\left(\log _{2} N_{k}\right) \sqrt{\log _{2} \log _{2} N_{k}}}$, for all $k \geq 1$.

Proof. Pick any $A \subset S_{k} \cup S_{k+1}$ such that $|A|=N_{k}$ and $\left|A \cap S_{k}\right|=\left|A \cap S_{k+1}\right|=N_{k} / 2$. Then

$$
\left\|\mathbf{1}_{A}\right\| \geq \alpha_{k} N_{k} / 2=\frac{N_{k} / 2}{\log _{2} N_{k}}
$$

Next, pick $B=S_{k}$, so that $|B|=|A|=N_{k}$ and

$$
\left\|\mathbf{1}_{B}\right\|=\max \left\{1, \alpha_{k} \cdot 0, \sup _{n \leq k-1} \beta_{n} N_{n}\right\}=\beta_{k-1} N_{k-1}=\sqrt{N_{k-1}}=\sqrt{\log _{2} \log _{2} N_{k}} .
$$

Then $\mu_{N_{k}} \geq\left\|\mathbf{1}_{A}\right\| /\left\|\mathbf{1}_{B}\right\| \geq \frac{N_{k} / 2}{\left(\log _{2} N_{k}\right) \sqrt{\log _{2} \log _{2} N_{k}}}$.

Claim 2: $\quad \mu_{N_{k}}^{d} \leq \tilde{\mu}_{N_{k}}^{d} \leq \sqrt{N_{k}}$, for all $k \geq 2$.

Proof. Let $A, B$ be any pair of disjoint sets with $|A|=|B| \leq N_{k}$, and let $|\varepsilon|=|\eta|=1$. If $|A|=|B| \leq \sqrt{N_{k}}$, then the trivial bounds $\left\|\mathbf{1}_{\varepsilon A}\right\| \leq|A|$ and $\left\|\mathbf{1}_{\eta B}\right\| \geq 1$ give

$$
\frac{\left\|\mathbf{1}_{\varepsilon A}\right\|}{\left\|\mathbf{1}_{\eta B}\right\|} \leq \sqrt{N_{k}} .
$$

So, it remains to consider the cases $\sqrt{N_{k}}<|A|=|B| \leq N_{k}$. We split $A$ into three parts

$$
A_{0}=A \cap S_{k}, \quad A_{+}=A \cap T_{k}, \quad A_{-}=A \cap\left[S_{1} \cup \ldots \cup S_{k-1}\right] .
$$

Then, we have the following upper bound

$$
\begin{aligned}
\left\|\mathbf{1}_{\varepsilon A}\right\| & \leq \max \left\{1, \sup _{n<k} \alpha_{n}\left|A_{-}\right|, \alpha_{k}\left|A_{0}\right|, \sup _{n>k} \alpha_{n} N_{k}, \sup _{n<k} \beta_{n} N_{n}, \sup _{n \geq k} \beta_{n}|A|\right\} \\
& \leq \max \left\{N_{k}^{\prime}, \alpha_{k}\left|A_{0}\right|, \beta_{k}|A|\right\},
\end{aligned}
$$

due to the elementary inequalities

- $\sup _{n<k} \alpha_{n}\left|A_{-}\right| \leq\left|A_{-}\right| \leq N_{k}^{\prime}$

- $\sup _{n>k} \alpha_{n} N_{k}=\alpha_{k+1} N_{k}=N_{k} 2^{-N_{k}} \leq 1$

- $\sup _{n<k} \beta_{n} N_{n}=\sqrt{N_{k-1}} \leq N_{k-1} \leq N_{k}^{\prime}$

- $\sup _{n \geq k} \beta_{n}|A|=\beta_{k}|A|$.

Moreover, since $\beta_{k}|A| \leq \min \left\{\beta_{k} N_{k}=\sqrt{N_{k}}, \alpha_{k}|A|\right\}$, we derive

$$
\left\|\mathbf{1}_{\varepsilon A}\right\| \leq \max \left\{\sqrt{N_{k}}, \alpha_{k}\left|A_{0}\right|\right\} \quad \text { and } \quad\left\|\mathbf{1}_{\varepsilon A}\right\| \leq \max \left\{N_{k}^{\prime}, \alpha_{k}|A|\right\} .
$$

We now give a lower bound for $\left\|\mathbf{1}_{\eta B}\right\|$. The key estimate will rely on the following

Lemma 5.5. Let $B_{0}=B \cap S_{k}$ and $B_{0}^{c}=S_{k} \backslash B_{0}$. Then

$$
\sup _{\sigma \in \mathfrak{N}_{k}}\left|\left\langle\mathbf{1}_{\sigma S_{k}}, \mathbf{1}_{\eta B_{0}}\right\rangle\right| \geq \min \left\{\left|B_{0}\right|,\left|B_{0}^{c}\right|\right\}
$$


Proof. If $\left|B_{0}\right| \leq N_{k} / 2$, then we may select any $\sigma \in \mathfrak{N}_{k}$ such that $\left.\sigma\right|_{B_{0}}=\eta$ (which is possible since $\left.\left|B_{0}^{c}\right| \geq\left|B_{0}\right|\right)$, which gives

$$
\left|\left\langle\mathbf{1}_{\sigma S_{k}}, \mathbf{1}_{\eta B_{0}}\right\rangle\right|=\left|B_{0}\right|=\min \left\{\left|B_{0}\right|,\left|B_{0}^{c}\right|\right\} .
$$

Assume now that $\left|B_{0}\right|>N_{k} / 2$. Pick any $S \subset B_{0}$ with $|S|=\left|B_{0}^{c}\right|=N_{k}-\left|B_{0}\right|$. Choose $v \in\{-1,1\}^{B_{0}^{c}}$ so that $\sum_{i \in S} \eta_{i}+\sum_{i \in B_{0}^{c}} v_{i}=0$. Choose $\tau \in\{-1,1\}^{B_{0} \backslash S}$ so that $\sum_{i \in B_{0} \backslash S} \tau_{i}=0$. Replacing $\tau$ by $-\tau$, if necessary, we may assume that $\sum_{i \in B_{0} \backslash S} \tau_{i} \eta_{i} \geq 0$. Finally, define $\sigma \in \mathfrak{N}_{k}$ by setting

$$
\left.\sigma\right|_{S}=\left.\eta\right|_{S},\left.\quad \sigma\right|_{B_{0}^{c}}=\left.v\right|_{B_{0}^{c}},\left.\quad \sigma\right|_{B_{0} \backslash S}=\left.\tau\right|_{B_{0} \backslash S}
$$

Then,

$$
\left|\left\langle\mathbf{1}_{\sigma S_{k}}, \mathbf{1}_{\eta B_{0}}\right\rangle\right|=\sum_{i \in S} \eta_{i}^{2}+\sum_{i \in B_{0} \backslash S} \tau_{i} \eta_{i} \geq|S|=\left|B_{0}^{c}\right|=\min \left\{\left|B_{0}\right|,\left|B_{0}^{c}\right|\right\}
$$

From the lemma and the definition of the norm we see that

$$
\left\|\mathbf{1}_{\eta B}\right\| \geq \max \left\{1, \alpha_{k} \min \left\{\left|B_{0}\right|,\left|B_{0}^{c}\right|\right\}, \beta_{k}\left|B_{+}\right|\right\} .
$$

We shall finally combine the estimates in (5.9) and (5.11) to establish Claim 2. We distinguish two cases

Case 1: $\min \left\{\left|B_{0}\right|,\left|B_{0}^{c}\right|\right\}=\left|B_{0}^{c}\right|$. Then, since $A_{0} \subset B_{0}^{c}$, we see that

$$
\alpha_{k}\left|A_{0}\right| \leq \alpha_{k}\left|B_{0}^{c}\right| \leq\left\|\mathbf{1}_{\eta B}\right\|
$$

and therefore the first estimate in (5.9) gives

$$
\frac{\left\|\mathbf{1}_{\varepsilon A}\right\|}{\left\|\mathbf{1}_{\eta B}\right\|} \leq \frac{\max \left\{\sqrt{N_{k}},\left\|\mathbf{1}_{\eta B}\right\|\right\}}{\left\|\mathbf{1}_{\eta B}\right\|} \leq \sqrt{N_{k}} .
$$

Case 2: $\min \left\{\left|B_{0}\right|,\left|B_{0}^{c}\right|\right\}=\left|B_{0}\right|$. Then, (5.11) reduces to

$$
\left\|\mathbf{1}_{\eta B}\right\| \geq \max \left\{\alpha_{k}\left|B_{0}\right|, \beta_{k}\left|B_{+}\right|\right\} \geq \beta_{k} \frac{\left|B_{0}\right|+\left|B_{+}\right|}{2}=\beta_{k} \frac{|B|-\left|B_{-}\right|}{2} \geq \beta_{k}|B| / 4,
$$

since $\left|B_{-}\right| \leq N_{k}^{\prime} \leq \sqrt{N_{k}} / 2 \leq|B| / 2$, if $k \geq 2$. Also, the second bound in (5.9) reads

$$
\left\|\mathbf{1}_{\varepsilon A}\right\| \leq \alpha_{k}|A|
$$

since $N_{k}^{\prime} \leq \sqrt{N_{k}} / \log _{2} N_{k}=\alpha_{k} \sqrt{N_{k}} \leq \alpha_{k}|A|$, if $k \geq 2$. Thus

$$
\frac{\left\|\mathbf{1}_{\varepsilon A}\right\|}{\left\|\mathbf{1}_{\eta B}\right\|} \leq \frac{\alpha_{k}|A|}{\beta_{k}|B| / 4}=\frac{4 \alpha_{k}}{\beta_{k}}=\frac{4 \sqrt{N_{k}}}{\log _{2} N_{k}} \leq \sqrt{N_{k}} .
$$

This establishes Claim 2.

From Claims 1 and 2 we now deduce that

$$
\frac{\mu_{N_{k}}}{\left[\tilde{\mu}_{N_{k}}^{d}\right]^{2-\varepsilon}} \geq \frac{N_{k}^{\varepsilon / 2} / 2}{\left(\log _{2} N_{k}\right) \sqrt{\log _{2} \log _{2} N_{k}}} \rightarrow \infty,
$$

and therefore

$$
\limsup _{N \rightarrow \infty} \frac{\mu_{N}}{\left[\mu_{N}^{d}\right]^{2-\varepsilon}}=\limsup _{N \rightarrow \infty} \frac{\tilde{\mu}_{N}}{\left[\tilde{\mu}_{N}^{d}\right]^{2-\varepsilon}}=\infty
$$




\section{NORM CONVERGENCE OF $\mathfrak{C G}_{m}^{t} x$ AND $\mathscr{G}_{m}^{t} x$}

In this section we search for conditions in $\mathscr{B}=\left\{\mathbf{e}_{n}\right\}_{n=1}^{\infty}$ under which it holds

$$
\left\|x-\mathfrak{C G}_{m}(x)\right\| \rightarrow 0, \quad \forall x \in \mathbb{X} .
$$

In [18, Theorem 1.1] this convergence is asserted for all "bases" $\left\{\mathbf{e}_{n}, \mathbf{e}_{n}^{*}\right\}_{n=1}^{\infty}$ satisfying (a)(b)-(c). The proof however, does not seem complete, so we investigate here whether (6.1) may be true in that generality.

The solution to this question requires the notion of strong M-basis; see [20, Def 8.4]. We say that $\mathscr{B}$ is a strong M-basis if additionally to the conditions (a)-(d) in $\S 1$ it also holds

$$
\overline{\operatorname{span}\left\{\mathbf{e}_{n}\right\}_{n \in A}}=\{x \in \mathbb{X}: \operatorname{supp} x \subset A\}, \quad \forall A \subset \mathbb{N} .
$$

Clearly, all Schauder or Cesàro bases (in some ordering) are strong M-bases; see e.g. [17] for further examples. However, there exist M-bases which are not strong M-bases, see e.g. [20, p. 244], or [10] for seminormalized examples in Hilbert spaces.

Lemma 6.1. If $\mathscr{B}$ is an $M$-basis which is not a strong $M$-basis, then there exists an $x_{0} \in \mathbb{X}$ such that, for all Chebyshev greedy operators $\mathfrak{C G}_{m}$,

$$
\liminf _{m \rightarrow \infty}\left\|x_{0}-\mathfrak{C G}_{m} x_{0}\right\|>0 .
$$

Proof. If $\mathscr{B}$ is not a strong M-basis there exists some set $A \subset \mathbb{N}$ (necessarily infinite) and some $x_{0} \in \mathbb{X}$ with supp $x_{0} \subset A$ such that

$$
\boldsymbol{\delta}=\operatorname{dist}\left(x_{0},\left[\mathbf{e}_{n}\right]_{A}\right)>0 .
$$

Since supp $\mathfrak{C G}_{m} x_{0}$ is always a subset of $A$, this implies (6.3).

Remark 6.2. The above reasoning also implies that $\liminf _{m}\left\|x_{0}-\mathscr{G}_{m} x_{0}\right\|>0$, for all greedy operators $\mathscr{G}_{m}$. In particular, for M-bases which are not strong, the quasi-greedy condition

$$
C_{q}:=\sup _{\substack{\mathscr{S}_{m} \in \mathbb{G}_{m} \\ m \in \mathbb{N}}}\left\|\mathscr{G}_{m}\right\|<\infty
$$

does not imply that $\mathscr{G}_{m} x$ converges to $x$ for all $x \in \mathbb{X}$. So the standard characterization in [27, Theorem 1] needs the extra assumption that $\mathscr{B}$ is a strong M-basis.

A corrected version of [18, Theorem 1.1] (and also of " $3 \Rightarrow 1$ " in [27, Theorem 1]) is the following.

Proposition 6.3. If $\mathscr{B}$ is a strong $M$-basis then, for all Chebyshev t-greedy operators $\mathfrak{C} \mathfrak{G}_{m}^{t}$,

$$
\lim _{m \rightarrow \infty}\left\|x-\mathfrak{C} \mathfrak{G}_{m}^{t} x\right\|=0, \quad \forall x \in \mathbb{X} .
$$

If additionally $C_{q}<\infty$, then for all $t$-greedy operators $\mathscr{G}_{m}^{t}$,

$$
\lim _{m \rightarrow \infty}\left\|x-\mathscr{G}_{m}^{t} x\right\|=0, \quad \forall x \in \mathbb{X} .
$$

Proof. Given $x \in \mathbb{X}$ and $\varepsilon>0$, by (6.2) there exists $z=\sum_{n \in B} b_{n} \mathbf{e}_{n}$ such that $\|x-z\|<\varepsilon$, for some finite set $B \subset \operatorname{supp} x$. Let $\alpha=\min _{n \in B}\left|\mathbf{e}_{n}^{*}(x)\right|$ and

$$
\bar{\Lambda}_{\alpha}=\left\{n:\left|\mathbf{e}_{n}^{*}(x)\right| \geq \alpha\right\} \text {. }
$$

\footnotetext{
${ }^{1}$ We thank V. Kadets for kindly providing this reference.
} 
Since $\alpha>0$, this is a finite greedy set for $x$ which contains $B$. Moreover, we claim that

$$
\bar{\Lambda}_{\alpha} \subset \operatorname{supp} \mathfrak{C G}_{m}^{t} x=: A, \quad \forall m>\left|\bar{\Lambda}_{t \alpha}\right| .
$$

Indeed, if this was not the case there would exist $n_{0} \in \bar{\Lambda}_{\alpha} \backslash A$, and since $A$ is a $t$-greedy set for $x$, then $\min _{n \in A}\left|\mathbf{e}_{n}^{*}(x)\right| \geq t\left|\mathbf{e}_{n_{0}}^{*}(x)\right| \geq t \alpha$. So, $A \subset \bar{\Lambda}_{t \alpha}$, which is a contradiction since $m=|A|>\left|\bar{\Lambda}_{t \alpha}\right|$. Therefore, (6.7) holds and hence

$$
\left\|x-\mathfrak{C G}_{m}^{t} x\right\| \leq\left\|x-\sum_{n \in B} b_{n} \mathbf{e}_{n}\right\|<\varepsilon, \quad \forall m>\left|\bar{\Lambda}_{t \alpha}\right| .
$$

This establishes (6.5).

We now prove (6.6). As above, let $z=\sum_{n \in B} b_{n} \mathbf{e}_{n}$ with $B \subset \operatorname{supp} x$ and $\|x-z\|<\varepsilon$. Performing if necessary a small perturbation in the $b_{n}$ 's, we may assume that $b_{n} \neq \mathbf{e}_{n}^{*}(x)$ for all $n \in B$. Let now

$$
\alpha_{1}=\min _{n \in B}\left|\mathbf{e}_{n}^{*}(x)\right|, \quad \alpha_{2}=\min _{n \in B}\left|\mathbf{e}_{n}^{*}(x-z)\right|, \quad \text { and } \quad \alpha=\min \left\{\alpha_{1}, \alpha_{2}\right\}>0 .
$$

Consider the sets

$$
\bar{\Lambda}_{t \alpha}=\left\{n:\left|\mathbf{e}_{n}^{*}(x)\right| \geq t \alpha\right\}=\left\{n:\left|\mathbf{e}_{n}^{*}(x-z)\right| \geq t \alpha\right\},
$$

which for all $t \in(0,1]$ are greedy sets for both $x$ and $x-z$, and contain $B$. We claim that,

$$
\text { if } m>\left|\bar{\Lambda}_{t \alpha}\right| \text { and } A:=\operatorname{supp} \mathscr{G}_{m}^{t} x \text {, then } \bar{\Lambda}_{\alpha} \subset A \text { and } A \in G(x-z, m, t) \text {. }
$$

The assertion $\bar{\Lambda}_{\alpha} \subset A$ is proved exactly as in (6.7). Next, we must show that

$$
\text { if } n \in A \quad \text { then } \quad\left|\mathbf{e}_{n}^{*}(x-z)\right| \geq t \max _{k \notin A}\left|\mathbf{e}_{k}^{*}(x-z)\right|=t \max _{k \notin A}\left|\mathbf{e}_{k}^{*}(x)\right| .
$$

This is clear if $n \in A \backslash B$ since $\mathbf{e}_{n}^{*}(x-z)=\mathbf{e}_{n}^{*}(x)$, and $A \in G(x, m, t)$. On the other hand, if $n \in B$, then $\left|\mathbf{e}_{n}^{*}(x-z)\right| \geq \alpha_{2} \geq \alpha \geq \max _{k \in A^{c}}\left|\mathbf{e}_{k}^{*}(x)\right|$, the last inequality due to $\bar{\Lambda}_{\alpha} \subset A$. Thus (6.8) holds true, and therefore

$$
\mathscr{G}_{m}^{t}(x)-z=\sum_{n \in A} \mathbf{e}_{n}^{*}(x-z) \mathbf{e}_{n}=\overline{\mathscr{G}}_{m}^{t}(x-z)
$$

for some $\overline{\mathscr{G}}_{m}^{t} \in \mathbb{G}_{m}^{t}$. Thus,

$$
\left\|\mathscr{G}_{m}^{t}(x)-x\right\|=\left\|\left(I-\overline{\mathscr{G}}_{m}^{t}\right)(x-z)\right\| \leq\left(1+\left\|\overline{\mathscr{G}}_{m}^{t}\right\|\right) \varepsilon
$$

and the result follows from $\sup _{m}\left\|\overline{\mathscr{G}}_{m}^{t}\right\| \leq\left(1+4 C_{q} / t\right) C_{q}$, by [9, Lemma 2.1].

\section{REFERENCES}

[1] F. Albiac and J.L. Ansorena, Characterization of 1-almost greedy bases. Rev. Matem. Compl. 30 (1) (2017), 13-24.

[2] P. M. Berná, Equivalence between almost-greedy and semi-greedy bases. J. Math. Anal. Appl. 417 (2019), 218-225.

[3] P. M. Berná, Ó. Blasco, Characterization of greedy bases in Banach spaces. J. Approx. Theory 215 (2017), 28-39.

[4] P. M. BERnÁ, Ó. Blasco, G. GARRIGós, Lebesgue inequalities for the greedy algorithm in general bases. Rev. Mat. Complut. 30 (2017), 369-392.

[5] P. M. Berná, Ó. Blasco, G. Garrigós, E. Hernández, T. Oikhberg, Embeddings and Lebesgue-Type Inequalities for the Greedy Algorithm in Banach Spaces. Constr. Approx. 48 (3) (2018), 415-451.

[6] Z. Ciesielski, A bounded orthonormal system of polygonals. Studia Math. 31 (1968) 339-346. 
[7] S.J. Dilworth, N.J. Kalton, D. Kutzarova, On the existence of almost greedy bases in Banach spaces, Studia Math. 159 (1) (2003), 67-101.

[8] S.J. Dilworth, N.J. Kalton, D. Kutzarova, and V.N. Temlyakov, The Thresholding Greedy Algorithm, Greedy Bases, and Duality, Constr. Approx. 19, (2003), 575-597.

[9] S.J. Dilworth, D. Kutzarova, T. OikhberG, Lebesgue constants for the weak greedy algorithm, Rev. Matem. Compl. 28 (2) (2015), 393-409.

[10] L. N. Dovbysh, N. K. Nikolskit, V. N. Sudakov, How good can a nonhereditary family be? J. Sov. Math. 34 (6) (1986), 2050-2060.

[11] G. Garrigós, E. Hernández And T. OIKhberg, Lebesgue-type inequalities for quasi-greedy bases, Constr. Approx. 38 (3) (2013), 447-470.

[12] P. Hajek, V. Montesinos Santalucía, J. VanderwerfF, V. Zizler, Biorthogonal systems in Banach spaces. Springer-Verlag 2008.

[13] Y. Katznelson, An introduction to Harmonic Analysis, 2nd ed. Dover Publ Inc, New York, 1976.

[14] S.V. Konyagin And V.N. Temlyakov, A remark on greedy approximation in Banach spaces, East. J. Approx. 5 (1999), 365-379.

[15] S.V. KONYAGIN AND V.N. TEMLYAKOV, Greedy approximatin with regard to bases and general minimal systems, Serdica Math. J. 28 (2002), 305-328.

[16] S. RopelA, Properties of bounded orthogonal spline bases. In Approximation theory (Papers, VIth Semester, Stefan Banach Internat. Math. Center, Warsaw, 1975), pp. 197-205, Banach Center Publ., 4, Warsaw, 1979.

[17] W.H. RUCKLE, On the classification of biorthogonal sequences. Canadian J. Math 26 (1974), 721-733.

[18] C. SHAO, P. YE, Lebesgue constants for Chebyshev thresholding greedy algorithms, Journal of Inequalities and Applications (2018), Paper No. 102, 23 pp.

[19] I. SingER. Bases in Banach spaces I. Springer-Verlag, 1970.

[20] I. SINGER. Bases in Banach spaces II. Springer-Verlag, 1981.

[21] V. N. Temlyakov, Greedy algorithm and n-term trigonometric approximation, Const.Approx. 14 (1998), 569-587.

[22] V.N. Temlyakov, Greedy approximation. Cambridge University Press, 2011.

[23] V. N. Temlyakov, The best m-term approximation and greedy algorithms, Adv. Comput. 8 (1998), 249-265.

[24] V. N. Temlyakov, Sparse approximation with bases. Ed. by S. Tikhonov. Advanced Courses in Mathematics. CRM Barcelona. Birkhäuser-Springer, 2015.

[25] V. N. Temlyakov, M. YANG, P. YE, Lebesgue-type inequalities for greedy approximation with respect to quasi-greedy bases, East J. Approx 17 (2011), 127-138.

[26] F. WeIsz, On the Fejér means of bounded Ciesielski systems. Studia Math. 146 (3) (2001), $227-243$.

[27] P. WojtaszczyK, Greedy Algorithm for General Biorthogonal Systems, Journal of Approximation Theory, 107, (2000), 293-314. 
Pablo M. Berná, Departamento de Matemáticas, Universidad Autónoma de Madrid, 28049 MADRID, SPAIN

E-mail address: pablo.berna@uam.es

Óscar Blasco, Department of Analysis Mathematics, Universidad de Valencia, CamPUS DE BURJASSOT, VALENCIA, 46100, SPAIN

E-mail address: oscar.blasco@uv.es

Gustavo Garrigós, Departamento de Matemáticas, Universidad de Murcia, 30100 MurCIA, SPAIN

E-mail address: gustavo.garrigos@um.es

Eugenio Hernández, Departamento de Matemáticas, Universidad Autónoma de Madrid, 28049 MADRID, SPAIN

E-mail address: eugenio.hernandez@uam.es

Timur Oikhberg, Department of Mathematics, University of Illinois Urbana-Champaign, URBANA, IL 61807, USA

E-mail address: oikhberg@illinois.edu 